\title{
Study of CS, SiO, and SiS abundances in carbon star envelopes: assessing their role as gas-phase precursors of dust ${ }^{\star, \star \star}$
}

\author{
S. Massalkhi, M. Agúndez, and J. Cernicharo
}

\author{
Instituto de Física Fundamental, CSIC, C/Serrano 123, 28006 Madrid, Spain \\ e-mail: sarah.massalkhi@csic.es
}

Received 16 January 2019 / Accepted 24 May 2019

\begin{abstract}
Aims. We aim to determine the abundances of $\mathrm{CS}, \mathrm{SiO}$, and $\mathrm{SiS}$ in a large sample of carbon star envelopes covering a wide range of mass loss rates to investigate the potential role that these molecules could play in the formation of dust in the surroundings of the central AGB star.

Methods. We surveyed a sample of 25 carbon-rich AGB stars in the $\lambda 2 \mathrm{~mm}$ band, more concretely in the $J=3-2$ line of CS and $\mathrm{SiO}$, and in the $J=7-6$ and $J=8-7$ lines of SiS, using the IRAM $30 \mathrm{~m}$ telescope. We performed excitation and radiative transfer calculations based on the large velocity gradient (LVG) method to model the observed lines of the molecules and to derive their fractional abundances in the observed envelopes. We also assessed the effect of infrared pumping in the excitation of the molecules. Results. We detected CS in all 25 targeted envelopes, $\mathrm{SiO}$ in 24 of them, and $\mathrm{SiS}$ in 17 sources. Remarkably, SiS is not detected in any envelope with a mass loss rate below $10^{-6} M_{\odot} \mathrm{yr}^{-1}$ while it is detected in all envelopes with mass loss rates above that threshold. We found that CS and SiS have similar abundances in carbon star envelopes, while SiO is present with a lower abundance. We also found a strong correlation in which the denser the envelope, the less abundant are CS and SiO. The trend is however only tentatively seen for $\mathrm{SiS}$ in the range of high mass loss rates. Furthermore, we found a relation in which the integrated flux of the $\mathrm{MgS}$ dust feature at $30 \mu \mathrm{m}$ increases as the fractional abundance of CS decreases.

Conclusions. The decline in the fractional abundance of CS with increasing density could be due to gas-phase chemistry in the inner envelope or to adsorption onto dust grains. The latter possibility is favored by a correlation between the CS fractional abundance and the $30 \mu \mathrm{m}$ feature, which suggests that CS is efficiently incorporated onto MgS dust around C-rich AGB stars. In the case of SiO, the observed abundance depletion with increasing density is most likely caused by an efficient incorporation onto dust grains. We conclude that $\mathrm{CS}, \mathrm{SiO}$ (very likely), and $\mathrm{SiS}$ (tentatively) are good candidates to act as gas-phase precursors of dust in C-rich AGB envelopes.
\end{abstract}

Key words. astrochemistry - molecular processes - stars: abundances - stars: AGB and post-AGB - circumstellar matter

\section{Introduction}

The circumstellar envelopes (CSEs) of asymptotic giant branch (AGB) stars, formed through extensive stellar mass loss, are rich in chemical diversity and have long been known to be efficient sites for molecule and grain formation. In the Galaxy, AGB stars are the main sources of interstellar dust (Gehrz 1989). It is therefore of particular interest to study their CSEs because these envelopes contain gas (mainly in the form of molecules), dust, and products of nucleosynthesis that are eventually expelled into the interstellar medium (ISM) and will contribute to its evolution and enrichment (Herwig 2005).

The type of molecules and dust grains found in CSEs is to a great extent determined by the carbon-to-oxygen $(\mathrm{C} / \mathrm{O})$ abundance ratio at the photosphere of the AGB star. At the temperatures and densities of AGB atmospheres, thermochemical equilibrium (TE) predicts that carbon monoxide (CO) molecules have a very high abundance because of their stability, which locks most of the available carbon in oxygen-rich stars (M-type,

\footnotetext{
${ }^{\star}$ Reduced spectra (FITS) are available at the CDS via anonymous ftp to cdsarc.u-strasbg. fr $(130.79 .128 .5)$ or via http:// cdsarc.u-strasbg.fr/viz-bin/qcat? J/A+A/628/A62

$\star \star$ Based on observations carried out with the IRAM $30 \mathrm{~m}$ Telescope. The Institut de Radioastronomie Millimétrique (IRAM) is supported by INSU/CNRS (France), MPG (Germany) and IGN (Spain).
}

$\mathrm{C} / \mathrm{O}<1)$ or oxygen in carbon-rich stars (C-type, $\mathrm{C} / \mathrm{O}>1)$. As a consequence of this, envelopes around $\mathrm{M}$-type stars contain a variety of oxygen-bearing molecules (e.g., $\mathrm{H}_{2} \mathrm{O}, \mathrm{SiO}$, and TiO; see Velilla Prieto et al. 2017) and silicate dust, while CSEs around C-type stars display a variety of carbon-bearing molecules (e.g., $\mathrm{C}_{2} \mathrm{H}_{2}, \mathrm{HCN}, \mathrm{CS}$, and $\mathrm{SiC}_{2}$; see Olofsson et al. 1993 and Cernicharo et al. 2000) and contain carbonaceous, silicon carbide, and magnesium sulfide dust. After some gas-phase precursors condense near the surroundings of the stellar photosphere, the condensation nuclei grow to micrometer sizes as a consequence of accretion and coagulation processes. In C-rich AGB stars, molecules such as CS (Olofsson et al. 1993), SiS (Schöier et al. 2007), and SiO (Schöier et al. 2006b) are found to be abundant and are potential precursors of dust grains, in which case their abundances in the gas phase should experience a decline as they incorporate into solid grains. Eventually, interstellar ultraviolet (UV) photons destroy the molecules remaining in the gas phase in the outer envelope owing to photodissociation. However, the picture is yet poorly constrained from an observational point of view. It is still not well understood what are the gas-phase building blocks of dust grains in CSEs.

One of the first major studies of abundances in a large sample of AGB stars was performed by González Delgado et al. (2003), who investigated $\mathrm{SiO}$ in 40 M-type stars. Later on, Schöier et al. (2006b) studied $\mathrm{SiO}$ in a sample of 19 C-rich AGB 
Table 1. Sample of carbon stars.

\begin{tabular}{|c|c|c|c|c|c|c|c|c|c|c|c|}
\hline Name & $\begin{array}{c}\text { RA } \\
\text { J2000.0 }\end{array}$ & $\begin{array}{c}\text { Dec } \\
\text { J2000.0 }\end{array}$ & $\begin{array}{c}V_{\mathrm{LSR}} \\
\left(\mathrm{km} \mathrm{s}^{-1}\right)\end{array}$ & $\begin{array}{c}D \\
(\mathrm{pc})\end{array}$ & $\begin{array}{l}T_{\star} \\
(\mathrm{K}) \\
\end{array}$ & $\begin{array}{c}L_{\star} \\
\left(L_{\odot}\right) \\
\end{array}$ & $\begin{array}{c}\dot{M} \\
\left(M_{\odot} \mathrm{yr}^{-1}\right)\end{array}$ & $\begin{array}{c}V_{\exp } \\
\left(\mathrm{km} \mathrm{s}^{-1}\right)\end{array}$ & $\begin{array}{c}T_{\mathrm{d}}\left(r_{\mathrm{c}}\right) \\
(\mathrm{K})\end{array}$ & $\begin{array}{c}r_{\mathrm{c}} \\
(\mathrm{cm})\end{array}$ & $\Psi$ \\
\hline $2 C+10216$ & $09: 47: 57.45$ & $+13: 16: 43.9$ & -26.5 & 130 & 2330 & 8750 & $2.0 \times 10^{-5}$ & 14.5 & 800 & $2.0 \times 10^{14}$ & 300 \\
\hline CIT 6 & $10: 16: 02.27$ & $+30: 34: 18.6$ & -1 & 400 & 1800 & 10000 & $6.0 \times 10^{-6}$ & 17 & 1000 & $2.1 \times 10^{14}$ & 141 \\
\hline CRL 3068 & $23: 19: 12.24$ & $+17: 11: 33.4$ & -31.5 & 1300 & 1800 & 10900 & $2.5 \times 10^{-5}$ & 14.5 & 1500 & $2.0 \times 10^{14}$ & 174 \\
\hline S Cep & $21: 35: 12.83$ & $+78: 37: 28.2$ & -15.3 & 380 & 2200 & 7300 & $1.2 \times 10^{-6}$ & 22.5 & 1400 & $5.8 \times 10^{13}$ & 360 \\
\hline $\mathrm{IRC}+30374$ & 19:34:09.87 & $+28: 04: 06.3$ & -12.5 & 1200 & 2000 & 9800 & $1.0 \times 10^{-5}$ & 25 & 1000 & $2.2 \times 10^{14}$ & 1008 \\
\hline Y CVn & $12: 45: 07.83$ & $+45: 26: 24.9$ & +22 & 220 & 2200 & 4400 & $1.5 \times 10^{-7}$ & 7 & 1500 & $8.7 \times 10^{13}$ & 500 \\
\hline LP And & $23: 34: 27.53$ & $+43: 33: 01.2$ & -17 & 630 & 1900 & 9600 & $7.0 \times 10^{-6}$ & 14.5 & 1100 & $1.8 \times 10^{14}$ & 288 \\
\hline V Cyg & $20: 41: 18.27$ & $+48: 08: 28.8$ & +13.5 & 366 & 2300 & 6000 & $1.6 \times 10^{-6}$ & 12 & 1400 & $9.4 \times 10^{13}$ & 364 \\
\hline UU Aur & $06: 36: 32.84$ & $+38: 26: 43.8$ & +6.7 & 260 & 2800 & 6900 & $2.4 \times 10^{-7}$ & 10.6 & 1500 & $6.3 \times 10^{13}$ & 1000 \\
\hline V384 Per & $03: 26: 29.51$ & $+47: 31: 48.6$ & -16.8 & 560 & 2000 & 8100 & & & 1300 & & 584 \\
\hline $\mathrm{IRC}+60144$ & $04: 35: 17.54$ & $+62: 16: 23.8$ & -48.8 & 1030 & 2000 & 7800 & $3.7 \times 10^{-6}$ & 19.5 & 1200 & $2.0 \times 10^{14}$ & 1014 \\
\hline U Cam & $03: 41: 48.17$ & $+62: 38: 54.4$ & +6 & 430 & 2695 & 7000 & $2.0 \times 10^{-7}$ & 13 & 1500 & $4.4 \times 10^{13}$ & 833 \\
\hline V636 Mon & $06: 25: 01.43$ & $-09: 07: 15.9$ & +10 & 880 & 2500 & 8472 & $5.8 \times 10^{-6}$ & 20 & 1200 & $1.7 \times 10^{14}$ & 300 \\
\hline $\mathrm{IRC}+20370$ & $18: 41: 54.39$ & $+17: 41: 08.5$ & -0.8 & 600 & 2200 & 7900 & $3.0 \times 10^{-6}$ & 14 & 1500 & $8.1 \times 10^{13}$ & 266 \\
\hline R Lep & $04: 59: 36.35$ & $-14: 48: 22.5$ & +11.5 & 432 & 2200 & 5500 & & 17.5 & 1000 & $1.8 \times 10^{14}$ & 500 \\
\hline W Ori & $05: 05: 23.72$ & $+01: 10: 39.5$ & -1 & 220 & 2600 & 3500 & $7.0 \times 10^{-8}$ & 11 & 1500 & $4.3 \times 10^{13}$ & 333 \\
\hline CRL 67 & $00: 27: 41.10$ & $+69: 38: 51.5$ & -27.5 & 1410 & 2500 & 9817 & $1.1 \times 10^{-5}$ & 16 & 1200 & $1.8 \times 10^{14}$ & 495 \\
\hline CRL 190 & $01: 17: 51.62$ & $+67: 13: 55.4$ & -39.5 & 2790 & 2500 & 16750 & $6.4 \times 10^{-5}$ & 17 & 1000 & $4.7 \times 10^{14}$ & 424 \\
\hline S Aur & $05: 27: 07.45$ & $+34: 08: 58.6$ & -17 & 300 & 3000 & 8900 & $4.0 \times 10^{-7}$ & 24.5 & 1500 & $7.3 \times 10^{13}$ & 500 \\
\hline V Aql & $19: 04: 24.15$ & $-05: 41: 05.4$ & +53.5 & 330 & 2800 & 6500 & $1.4 \times 10^{-7}$ & 8 & 1500 & $6.1 \times 10^{13}$ & 500 \\
\hline CRL 2513 & 20:09:14.25 & $+31: 25: 44.9$ & +17.5 & 1760 & 2500 & 8300 & $2.0 \times 10^{-5}$ & 25.5 & 1200 & $1.6 \times 10^{14}$ & 453 \\
\hline CRL 2477 & $19: 56: 48.43$ & $+30: 43: 59.9$ & +5 & 3380 & 3000 & 13200 & $1.1 \times 10^{-4}$ & 20 & 1800 & $2.8 \times 10^{14}$ & 532 \\
\hline CRL 2494 & 20:01:08.51 & $+40: 55: 40.2$ & +29 & 1480 & 2400 & 10200 & $7.5 \times 10^{-6}$ & 20 & 1200 & $2.3 \times 10^{14}$ & 436 \\
\hline RV Aqr & $21: 05: 51.74$ & $-00: 12: 42.0$ & +0.5 & 670 & 2200 & 6800 & $2.3 \times 10^{-6}$ & 15 & 1300 & $7.6 \times 10^{13}$ & 200 \\
\hline ST Cam & $04: 51: 13.35$ & $+68: 10: 07.6$ & -13.6 & 360 & 2800 & 4400 & $1.3 \times 10^{-7}$ & 8.9 & 1500 & $5.0 \times 10^{13}$ & 500 \\
\hline
\end{tabular}

Notes. The adopted parameters are discussed in Massalkhi et al. (2018). See references therein. $\Psi$ for UU Aur and R Lep is from Schöier \& Olofsson (2001) and for IRC +60144 from Groenewegen et al. (2002).

stars. Interestingly, it was found that $\mathrm{SiO}$ behaves similarly in both types of stars, showing a trend of decreasing abundance with increasing mass loss rate, thought to be due to an increased depletion of $\mathrm{SiO}$ onto dust grains. On the other hand, when Schöier et al. (2007) investigated SiS in a reduced sample of C-rich stars, they did not find a clear trend; this contrasted with the results of $\mathrm{SiO}$, which could imply that $\mathrm{SiS}$ is less likely to be adsorbed onto dust grains than $\mathrm{SiO}$ in carbon-rich envelopes. Recently, we investigated $\mathrm{SiC}_{2}$ in a sample of 25 carbon-rich AGB stars and found a similar trend as that found for $\mathrm{SiO}$; that is, we discovered an abundance decline with increasing envelope density, which points to $\mathrm{SiC}_{2}$ being efficiently incorporated into dust grains and playing an important role in the formation of silicon carbide dust (Massalkhi et al. 2018).

In this paper, we follow up on our last study to investigate the abundance of $\mathrm{CS}, \mathrm{SiO}$, and $\mathrm{SiS}$ in the envelopes of carbon stars and to understand their potential role as gas-phase precursors of dust grains. We present observations of $\mathrm{SiO}(J=3-2), \mathrm{SiS}(J=$ $7-6$ and $J=8-7)$, and CS $(J=3-2)$ in a sample of 25 carbon stars with diverse mass loss rates. We carried out a detailed nonlocal thermodynamic equilibrium (non-LTE) radiative transfer analysis to derive molecular abundances in the CSEs. The sample of stars and observational details are presented in Sect. 2 and the main results obtained from the observations in Sect. 3. In Sect. 4 we describe the model and the excitation and radiative transfer calculations and discuss the most interesting features from these calculations in Sect. 5. Finally, we discuss the main implications of our study in Sect. 6 and present our conclusions in Sect. 7.

\section{Observations}

The observations were carried out in September 2017 with the IRAM $30 \mathrm{~m}$ telescope, located at Pico Veleta, Spain. The sample of 25 C-rich AGB stars observed is the same used in our previous study of $\mathrm{SiC}_{2}$ (Massalkhi et al. 2018) and was selected according to intense molecular emission, mainly based on the intensity of the HCN $J=1-0$ line (Loup et al. 1993; Bujarrabal et al. 1994; Schöier et al. 2013). The observed sources and their parameters are listed in Table 1. In this study, we focused on the emission of $\mathrm{CS}, \mathrm{SiO}$, and $\mathrm{SiS}$ and therefore the spectral setup used was slightly shifted from that employed in Massalkhi et al. (2018) and accommodated in a way to include the lines CS $J=3-2$, $\mathrm{SiO} J=3-2$ and $\mathrm{SiS} J=7-6$ and $J=8-7$ (see line parameters in Table 2).

We used the E150 receiver in dual side band, with image rejections $>10 \mathrm{~dB}$, and observed the frequency ranges 125.7-133.5 GHz and 141.4-149.2 GHz in the lower and upper side bands, respectively. The beam size of the telescope at these frequencies is in the range 16.7-19.3". We used the wobblerswitching technique. This technique consists of a symmetric position switching with the OFF position (atmosphere) at $180^{\prime \prime}$ in azimuth from the $\mathrm{ON}$ position (source + atmosphere). Spectra at the OFF and ON positions are taken by nutating the secondary mirror at a rate of $0.5 \mathrm{~Hz}$, and the OFF is subtracted from the $\mathrm{ON}$ to remove the contribution of the atmosphere to the signal. The focus was regularly checked on Venus and the pointing of the telescope was systematically checked on a nearby quasar before observing each AGB star. The error in 
Table 2. Covered rotational transitions of $\mathrm{CS}, \mathrm{SiO}$, and SiS.

\begin{tabular}{lcccr}
\hline \hline Transition & $\begin{array}{c}\text { Frequency } \\
(\mathrm{MHz})\end{array}$ & $\begin{array}{c}A_{\mathrm{ul}} \\
\left(\mathrm{s}^{-1}\right)\end{array}$ & $\begin{array}{c}E_{\mathrm{u}} \\
(\mathrm{K})\end{array}$ & $\begin{array}{r}\theta_{\mathrm{mb}} \\
\left({ }^{\prime \prime}\right)\end{array}$ \\
\hline $\mathrm{CS} J=3-2$ & 146969.025 & $6.07 \times 10^{-5}$ & 14.1 & 16.7 \\
SiO $J=3-2$ & 130268.665 & $1.06 \times 10^{-4}$ & 12.5 & 18.8 \\
SiS $J=7-6$ & 127076.178 & $3.36 \times 10^{-5}$ & 24.4 & 19.3 \\
SiS $J=8-7$ & 145227.052 & $5.05 \times 10^{-5}$ & 31.4 & 16.9 \\
\hline
\end{tabular}

the pointing is estimated to be $2-3^{\prime \prime}$. The E150 receiver was connected to a fast Fourier transform spectrometer providing a spectral resolution of $0.2 \mathrm{MHz}$. The weather was good and stable during most of the observations, with typical amounts of precipitable water vapor of 1-3 $\mathrm{mm}$ and average system temperatures of $115 \mathrm{~K}$. The intensity scale, calibrated using two absorbers at different temperatures and the atmospheric transmission model (ATM) (Cernicharo 1985; Pardo et al. 2001), is expressed in terms of $T_{\mathrm{mb}}$, the main beam brightness temperature. The error in the intensities due to calibration is estimated to be $\sim 20 \%$.

The data were reduced using CLASS $^{1}$ within the package GILDAS $^{2}$. For each source, we averaged the spectra corresponding to the horizontal and vertical polarizations and subtracted a baseline consisting of a first order polynomial. When the lines were not very strong, the spectra were smoothed to a spectral resolution of $1 \mathrm{MHz}$ to increase the signal-to-noise ratio $(\mathrm{S} / \mathrm{N})$. This corresponds to a velocity resolution of $2-2.4 \mathrm{~km} \mathrm{~s}^{-1}$. Typical on source integration times, after averaging horizontal and vertical polarizations, were $\sim 1 \mathrm{~h}$ for each source, resulting in $T_{\mathrm{mb}} \mathrm{rms}$ noise levels per $1 \mathrm{MHz}$ channel of $2-6 \mathrm{mK}$.

\section{Observational results}

The spectra obtained are shown in Fig. 1 (CS $J=3-2)$, Fig. 2 ( $\mathrm{SiO} J=3-2$ ), and Figs. 3 and 4 ( $\mathrm{SiS} J=7-6$ and $J=$ $8-7$, respectively). The observed lines exhibit profiles typical of expanding circumstellar shells, i.e., either double-peaked, flattopped, or parabolic, which can be reasonably well fit by the shell method of CLASS. The method fits the function

$f(v)=\frac{A}{\Delta v} \frac{1+4 H\left[\left(v-v_{0}\right) / \Delta v\right]^{2}}{1+H / 3}$,

where $A$ is the area under the profile in $\mathrm{K} \mathrm{MHz}, v_{0}$ is the middle frequency in $\mathrm{MHz}, \Delta v$ is the full width at zero intensity level in $\mathrm{MHz}$, and $H$ is the horn-to-center ratio, which is dimensionless. The expansion velocity $V_{\exp }$ can then be obtained by the following expression:

$V_{\exp }=c \frac{\Delta v / 2}{v_{0}}$,

where $c$ is the speed of light.

By performing the fit, we aim to derive for the target lines in every source the centroid frequency, expansion velocity, and line area, i.e., the velocity-integrated intensity. Most of the observed lines show profiles that match one of the types mentioned above, apart from the two sources U Cam (see line profile of CS emission in Fig. 1) and CIT 6 (see line profile of $\mathrm{SiO}$ in Fig. 2), which have profiles that deviate from the expected line profile

\footnotetext{
1 Continuum and Line Analysis Single-dish Software.

2 http://www.iram. fr/IRAMFR/GILDAS
}

of a spherical expanding shell. The CS emission of UCam is interpreted below as having two components, which we faced no difficulty considering separately. CIT 6 shows a rather asymmetrical profile in the $\mathrm{SiO} J=3-2$, SiS $J=7-6$ and $J=8-7$ lines. In any case, the two most interesting parameters, the line area and the expansion velocity, are reasonably well fitted. The derived parameters of the observed line profiles are shown in Table A.1.

The observations resulted in the clear detection of CS $J=$ $3-2$ in all targeted sources. In the case of $\mathrm{SiO}$, we detected the $J=3-2$ line in all target sources with the exception of ST Cam. As previously mentioned, the case of U Cam deserves particular attention because this source exhibits emission arising from a present-day wind and from a geometrically thin detached shell surrounding the central star (Schöier et al. 2005). The detached shell is probably the result of episodic mass loss driven by helium shell flashes, i.e., thermal pulses (Olofsson et al. 1990). According to the observed line profiles, CS emission in U Cam arises from both the present-day wind and the detached envelope, while $\mathrm{SiO}$ emission arises exclusively from the present-day wind; compare line profiles of $\mathrm{CS}$ and $\mathrm{SiO}$ in Figs. 1 and 2, respectively, with that of CO $J=1-0$ in Fig. 6 of Schöier et al. (2005). To disentangle the contribution from each component in the emission line of CS, we fitted the observed line with two components of different line widths; the narrow line width corresponds to the present-day wind and the wide to the detached envelope. The green line in Fig. 1 corresponds to the emission from the present-day wind, which we are ultimately interested in modeling. In the case of SiS, the $J=7-6$ and $J=8-7$ lines were detected in 17 out of 25 sources, that is, in all sources except U Cam, V Aql, S Aur, W Ori, Y CVn, R Lep, ST Cam, and UU Aur.

The lines profile shapes usually give information about the emission being observed. When the emission is optically thick and unresolved by the beam of the telescope, the line profiles can be described as parabolic. We see that in most sources, the observed line shapes of $\mathrm{CS}$ and $\mathrm{SiO}$ exhibit such profile. If the emission is optically thin and unresolved, a flat-topped profile is seen. This kind of profile is seen in the observed lines of SiS. One notable exception for the three molecules is IRC +10216 , whose close proximity and high mass loss rate result in an extended envelope whose molecular emission is spatially resolved by the telescope beam, and the line profiles show a more or less marked double-peak character.

\section{Excitation and radiative transfer modeling}

We aim at deriving molecular abundances of CS, SiO, and SiS in each source of our sample to provide a global view of how abundant these molecules are in envelopes around carbon stars. For this purpose, we performed excitation and radiative transfer calculations. The rotational lines of $\mathrm{CS}, \mathrm{SiO}$, and $\mathrm{SiS}$ studied in this work have upper level energies in the range 12.5-31.4 K. As shown in Sect. 5, the emission from these lines does not arise from the inner parts of the envelope but from the intermediate and outer regions, where gas densities are not high enough to thermalize the rotational levels. Therefore, level populations cannot be described by local thermodynamic equilibrium (LTE), and detailed non-LTE excitation and radiative transfer calculations have to be carried out taking into account the specific parameters for each envelope (see Table 1) to derive accurate molecular abundances.

We consider an envelope model consisting of a central AGB star surrounded by a spherically symmetric envelope of gas and 

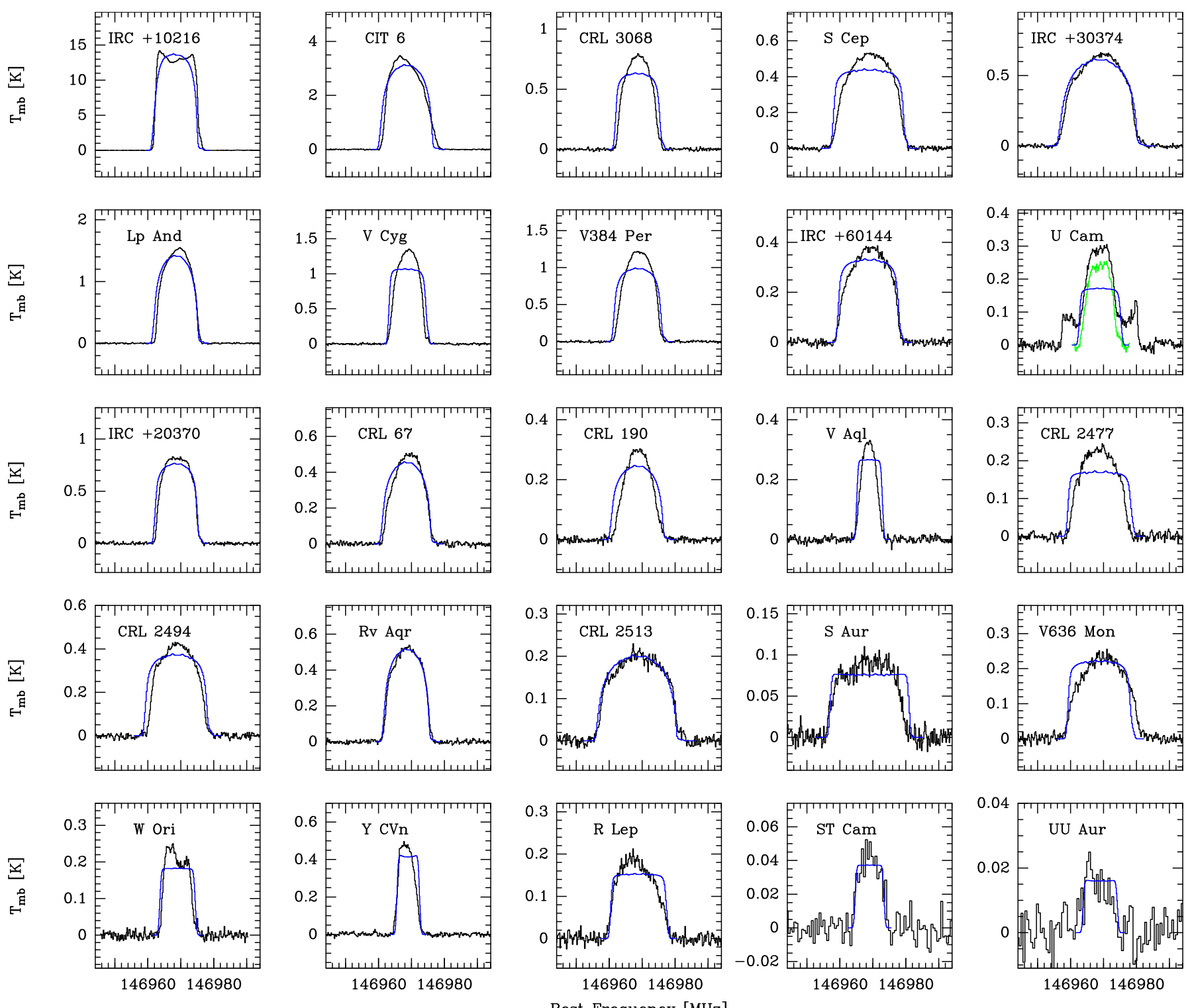

Fig. 1. CS $J=3-2$ line observed with the IRAM $30 \mathrm{~m}$ telescope in the 25 carbon stars (black histograms). U Cam shows emission contribution from the present day wind and the detached envelope. The green histogram corresponds to the observed line profile in which a fit to the detached envelope contribution (the wider one) has been subtracted. The blue lines indicate the calculated line profiles from the best-fit LVG model.

dust expanding at constant velocity $V_{\text {exp }}$ and with a mass loss rate $\dot{M}$, so that the radial density distribution follows an $r^{-2}$ law. The adopted physical properties of the stars and associated CSEs are presented in Table 1 . The various physical quantities describing the envelope, such as the radial profiles of the gas density, gas temperature, and dust temperature, as well as the properties of the dust grains, are described in Massalkhi et al. (2018).

We performed excitation and radiative transfer calculations to model the line emission of the studied molecules based on the multishell large velocity gradient (LVG) method. The LVG formalism is described in Sobolev (1960) and Castor (1970), whereas the implementation of the multishell method for CSEs is described in more detail in Agúndez (2009) and Agúndez et al. (2012). The LVG method deals well with optically thin lines and lines with moderate optical thickness (Castor 1970). This method is a good compromise with respect to other methodologies such as Monte Carlo, which are more computationally expensive and exhibit problems of convergence when including a high number of energy levels. Briefly, the circumstellar envelope is divided into a number of concentric shells, each of which has a characteristic set of physical properties. The excitation and radiative transfer is solved locally in each shell through statistical equilibrium equations, where collisional and radiative processes determine the level populations. In each shell, the contribution of the background radiation field is included and is composed of the cosmic microwave background, stellar radiation, and thermal emission from dust. We also include infrared (IR) pumping, that is, absorption of IR photons and pumping to excited vibrational states followed by spontaneous radiative decay to rotational levels in the ground vibrational state. This process has been found to play an important role in the excitation of some molecules in IRC +10216 (e.g., Agúndez \& Cernicharo 2006). For the three molecules studied here (CS, SiO, and $\mathrm{SiS}$ ), we only considered IR pumping to the first vibrationally excited state $(v=1)$. We did verify that adding upper vibrational states had no effect on the calculated line intensities, i.e., including up to $v=1$ changed 

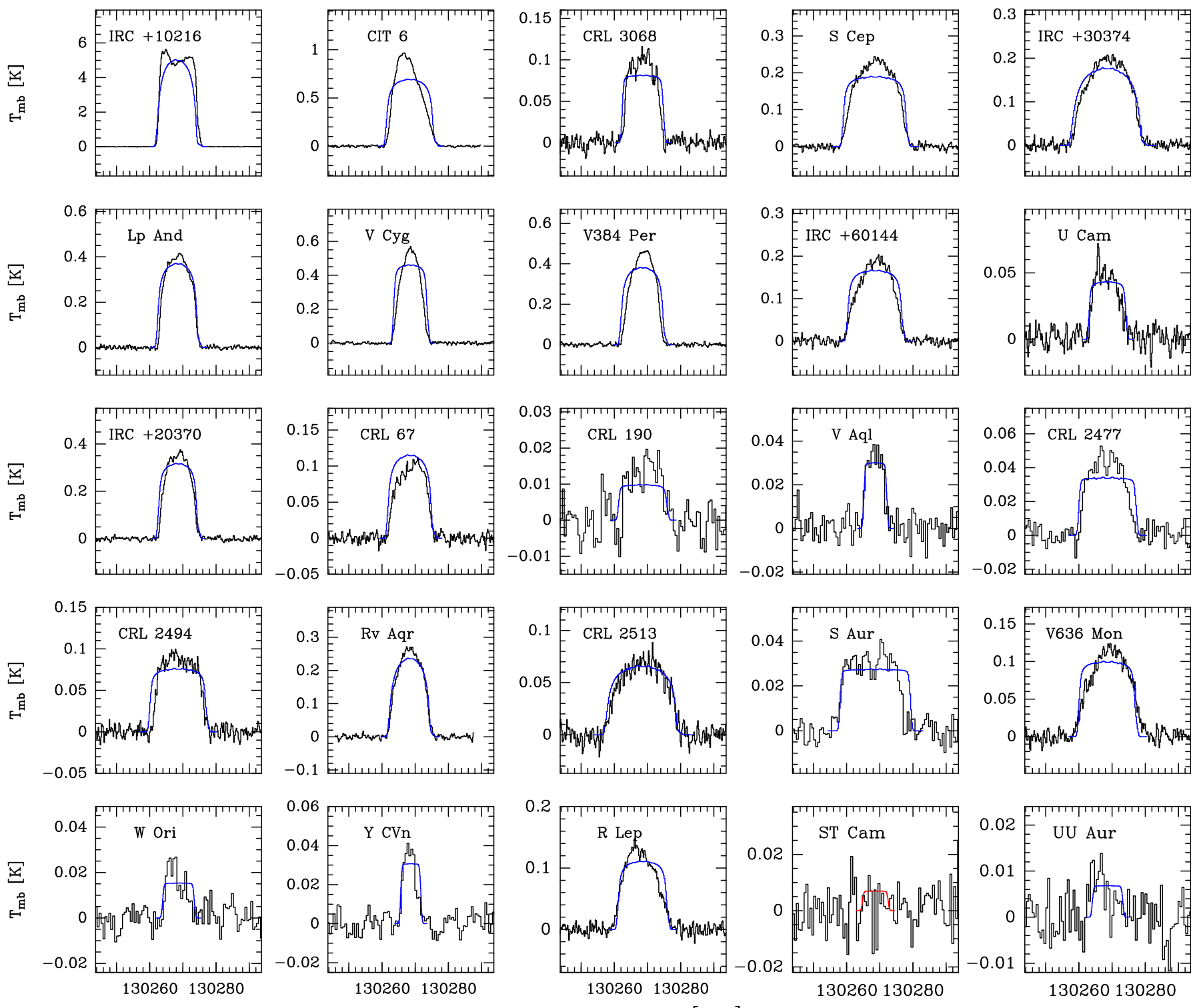

Fig. 2. SiO $J=3-2$ line observed with the IRAM $30 \mathrm{~m}$ telescope in the 25 carbon stars (black histograms). The blue lines indicate the calculated line profiles from the best-fit LVG model. The SiO line is not detected in ST Cam; the red line corresponds to the calculated line profile with the maximum intensity compatible with the non-detection.

the line intensities for the three molecules in all the stars, but including up to $v=3$ did not cause further changes.

\subsection{Molecular data}

A major prerequisite for a successful radiative transfer code is the availability of accurate spectroscopic and collisional excitation data. We discuss below the spectroscopic and collisional excitation data of the three molecules that were input into our calculations. We considered enough rotational states to include levels with energies higher than $2000 \mathrm{~K}$ to better deal with the inner hot regions of the envelopes.

In the excitation analysis of CS we considered the first 50 rotational levels within the $v=0$ and $v=1$ vibrational states (i.e., a total number of 100 energy levels). The level energies and transition frequencies were calculated from the Dunham coefficients given by Müller et al. (2005). The line strengths of pure rotational transitions were computed from the dipole moments for each vibrational state, $\mu_{v=0}=1.958 \mathrm{D}$ and $\mu_{v=1}=1.936 \mathrm{D}$
(Winnewisser \& Cook 1968), while for ro-vibrational transitions we used the Einstein coefficient of $15.8 \mathrm{~s}^{-1}$ given for the $v=$ $1 \rightarrow 0 \mathrm{P}(1)$ transition by Chandra et al. (1995). We adopted the collision rate coefficients recently calculated by Denis-Alpizar et al. (2018) for pure rotational transitions and up to temperatures of $300 \mathrm{~K}$. At higher temperatures and for ro-vibrational transitions we used the rate coefficients calculated by Lique \& Spielfiedel (2007) multiplying the original values computed for $\mathrm{He}$ as collider by the squared ratio of the reduced masses of the $\mathrm{H}_{2}$ and He colliding systems.

In the case of $\mathrm{SiO}$, we considered the first 50 rotational levels of the ground and first excited vibrational states. To calculate the line frequencies and strengths, we used the Dunham coefficients given by Sanz et al. (2003), the dipole moments for pure rotational transitions within the $v=0$ and $v=1$ vibrational states of 3.0982 and $3.1178 \mathrm{D}$, respectively, from Raymonda et al. (1970) and an Einstein coefficient for the ro-vibrational transition $v=1 \rightarrow 0 \mathrm{P}(1)$ of $6.61 \mathrm{~s}^{-1}$ from Drira et al. (1997). As collisional rate coefficients we adopted those calculated by 

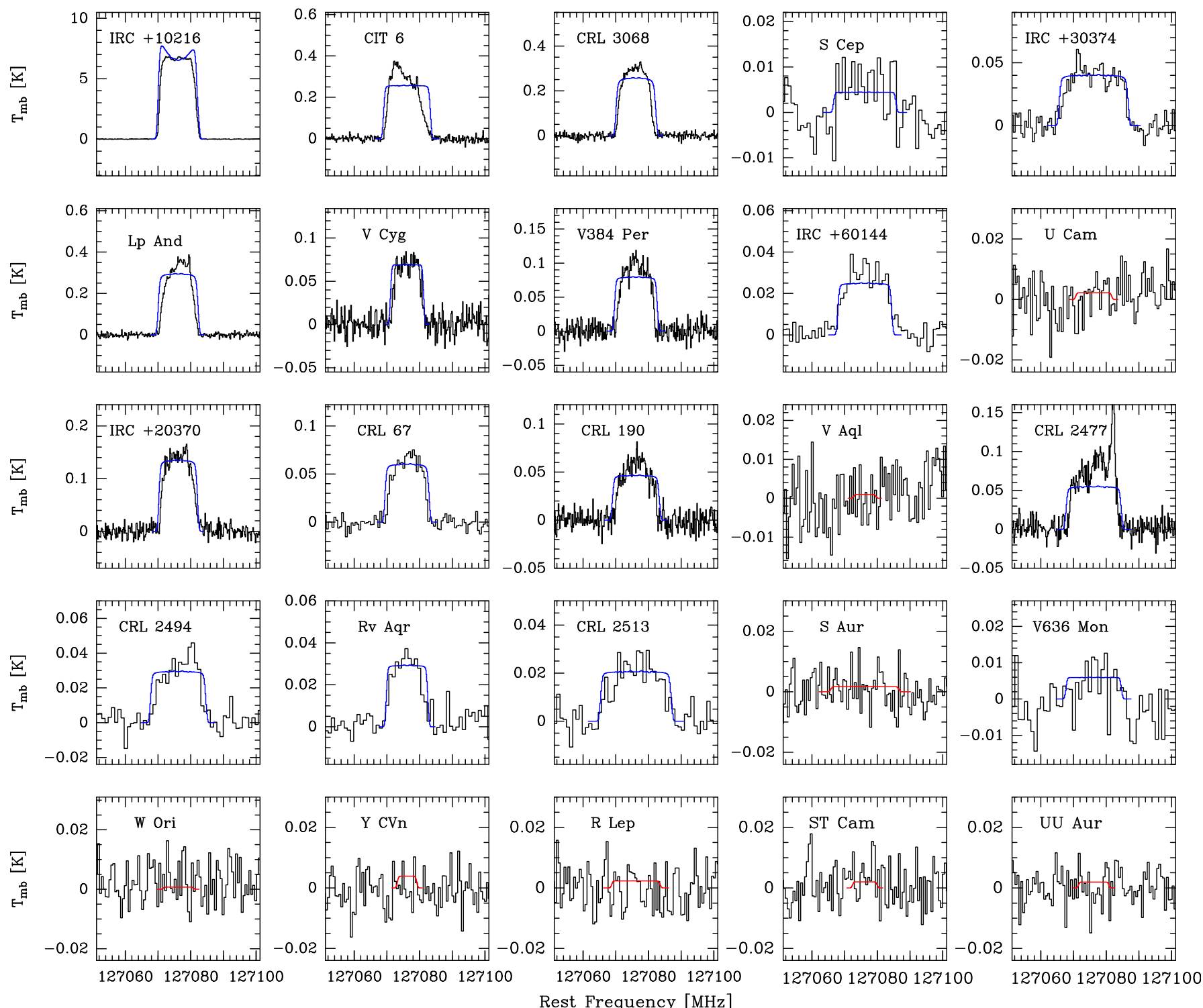

Fig. 3. SiS $J=7-6$ line observed with the IRAM $30 \mathrm{~m}$ telescope in the 25 carbon stars (black histograms). The blue lines indicate the calculated line profiles from the best-fit LVG model. The SiS line is not detected in various sources, for which we plot in red the calculated line profiles with the maximum intensity compatible with the non-detection.

Dayou \& Balança (2006) for pure rotational transitions and for temperatures up to $300 \mathrm{~K}$, while at higher temperatures and for ro-vibrational transitions we used the values computed by Balança \& Dayou (2017) scaling from $\mathrm{He}$ to $\mathrm{H}_{2}$ as collider as in the case of CS.

For $\mathrm{SiS}$, we include the first 70 rotational levels within the $v=0$ and $v=1$ vibrational states. Level energies were computed from the Dunham coefficients given by Müller et al. (2007). Line strengths were computed from the dipole moments $\mu_{v=0}=$ $1.735 \mathrm{D}, \mu_{v=1}=1.770 \mathrm{D}$, and $\mu_{v=1 \rightarrow 0}=0.13 \mathrm{D}$ (Müller et al. 2007 ; Piñeiro et al. 1987). The collisional rate coefficients have been taken from the calculations of Kłos \& Lique (2008), while for temperatures higher than $300 \mathrm{~K}$ and for ro-vibrational transitions we adopted the collisional rate coefficients computed by Toboła et al. (2008) scaled from $\mathrm{He}$ to $\mathrm{H}_{2}$ as with CS and $\mathrm{SiO}$.

\subsection{Abundance distributions}

We consider that $\mathrm{CS}, \mathrm{SiO}$, and $\mathrm{SiS}$ are formed close to the star with a given fractional abundance that remains constant throughout the envelope up to some region in the envelope where the abundance drops. This abundance falloff can be driven by at least two different processes: (1) condensation onto grains around the dust formation zone, and (2) photodissociation by the ambient UV radiation field in the outer envelope. While these molecules can certainly deplete in the dust formation region owing to condensation onto dust grains, in this work we are not sensitive to such potential abundance decline since the observed lines mostly probe intermediate and outer regions of the envelopes, that is, post-condensation regions (see Sect. 5). It is interesting to note that in IRC +10216 , the emission from CS, $\mathrm{SiO}$, and $\mathrm{SiS}$ vanishes at relatively outer radii, where photodissociation takes place (Bieging \& Nguyen-Quang-Rieu 1989; Lucas et al. 1992, 1995; Velilla Prieto et al. 2019). Various studies have reported on the abundance depletion in the inner regions of IRC +10216 . These studies show different degrees of depletion for the molecules, and in some cases the studies even have contradictory findings (Keady \& Ridgway 1993; Boyle et al. 1994; Schöier et al. 2006a; Decin et al. 2010a; Agúndez et al. 2012.) Regardless of whether these molecules may experience a first 

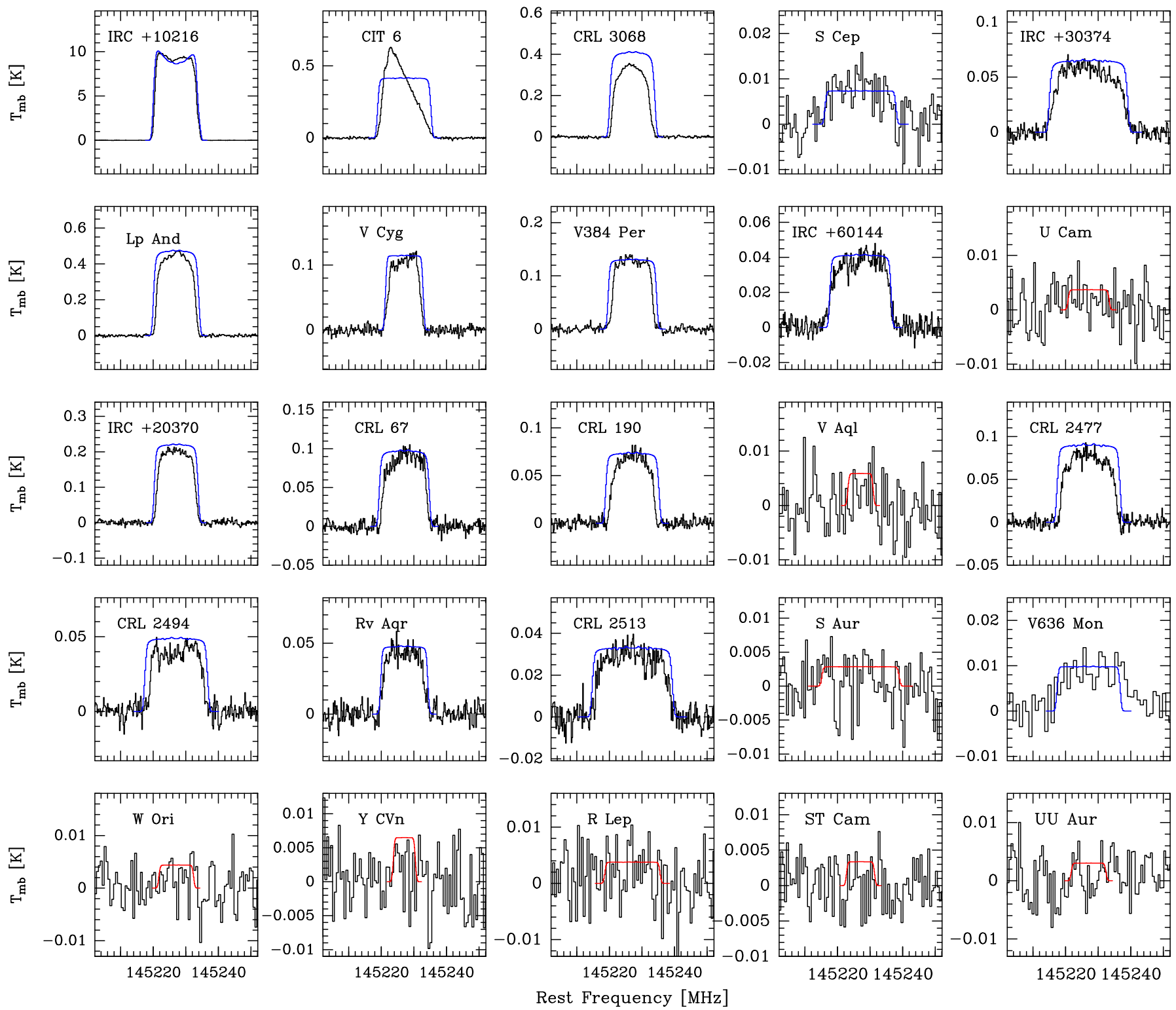

Fig. 4. Same as in Fig. 3 but for $\operatorname{SiS} J=8-7$.

abundance decline in the dust formation region or not, what is clear is that they maintain a significant abundance in the gas phase out to the outer envelope, where photodissociation is probably driving the disappearance of these molecules from the gas phase.

The above considerations suggest that a constant fractional abundance from the star and an abundance falloff driven by photodissociation in the outer envelope is a reasonable abundance distribution to model the lines observed in this work. In this scenario, the radial extent of each molecule would be entirely controlled by its corresponding photodissociation rate under the ambient UV radiation field and by the way in which circumstellar dust attenuates UV photons as they penetrate into the envelope. See Massalkhi et al. (2018) for more details on how the radial distributions were calculated using the photodissociation model. We however noticed that by using photodissociation rates from the literature when available (Heays et al. 2017 for $\mathrm{SiO}$ and Pattillo et al. 2018 for CS; see Sect. 6.4 for more details) and adopting the canonical interstellar $N_{\mathrm{H}} / A_{V}$ ratio for the local ISM (Bohlin et al. 1978), where $N_{\mathrm{H}}$ is the hydrogen column density in $\mathrm{cm}^{-2}$ and $A_{V}$ is the visual extinction measured in magnitudes, the radial extent of these molecules is very likely underestimated, at least for some of the envelopes. This suspicion was based on the fact that the abundances derived for $\mathrm{CS}$, $\mathrm{SiO}$, and/or $\mathrm{SiS}$ were anomalously high in some sources, as they exceeded the elemental abundances of sulfur and/or silicon, which a priori are expected to be similar to those in the Sun (Asplund et al. 2009). Given the small number of observed lines (one for CS and $\mathrm{SiO}$ and two for $\mathrm{SiS}$ ), the fact that they are sensitive to both the fractional abundance and the radial extent ${ }^{3}$ and the fact that the radial extent is very likely not well described by a simple photodissociation model, we thus decided to fix the radial extent using an empirical correlation from the literature (see below) and leave as a free parameter the fractional abundance so that it can be derived by modeling the observed lines. Therefore, following the work by González Delgado et al. (2003) and Schöier et al. (2006b, 2007), in this study we adopted a simple abundance

\footnotetext{
3 The emission arises from intermediate and outer regions of the envelopes and such regions are not resolved by the IRAM $30 \mathrm{~m}$ beam in most sources, with the notable exception of IRC +10216 .
} 
distribution given by a Gaussian

$f(r)=f_{0} \exp \left(-\left(r / r_{e}\right)^{2}\right)$

where $f$ is the fractional abundance relative to $\mathrm{H}_{2}, f_{0}$ is the initial abundance, and $r_{e}$ is the $e$-folding radius at which the abundance has dropped by a factor $e$. From a multiline study of $\mathrm{SiO}$ in M-type stars, González Delgado et al. (2003) found the following empirical correlation between the $e$-folding radius and the envelope density evaluated through the quantity $\dot{M} / V_{\text {exp }}$,

$\log r_{e}(\mathrm{SiO})=19.2+0.48 \log \left(\frac{\dot{M}}{V_{\mathrm{exp}}}\right)$,

where $r_{e}$ is given in cm, the mass loss rate $\dot{M}$ in $M_{\odot} \mathrm{yr}^{-1}$, and $V_{\text {exp }}$ in $\mathrm{km} \mathrm{s}^{-1}$. Although the scaling law in Eq. (4) was derived for $\mathrm{SiO}$ in oxygen-rich stars, we adopted this law for $\mathrm{SiO}$ and SiS in our sample of carbon stars. Schöier et al. (2006b, 2007) made the same assumption in the lack of better constraints for $\mathrm{C}$-type stars. Concerning the assumption of similar radial extents for $\mathrm{SiO}$ and $\mathrm{SiS}$, it is worth noting that although the photodissociation rate of $\mathrm{SiS}$ is unknown ${ }^{4}$, there are arguments that to a first order $\mathrm{SiO}$ and $\mathrm{SiS}$ should behave similarly with respect to photodissociation (van Dishoeck 1988; Wirsich 1994). Interferometric observations of these two molecules in IRC +10216 show that they have similar emission sizes, where the $\mathrm{SiO}$ emission is slightly more extended than that of SiS (Velilla Prieto et al. 2019). In the case of CS, we found it necessary to adopt a larger radial extent than for $\mathrm{SiO}$ and $\mathrm{SiS}$ because using Eq. (4) resulted in anomalously high CS abundances for some sources. We therefore adopted the following relation between $e$-folding radius and density in the envelope:

$\log r_{e}(\mathrm{CS})=19.65+0.48 \log \left(\frac{\dot{M}}{V_{\exp }}\right)$,

which was derived by starting from Eq. (4) and increasing the first term in small steps so that the amount of sulfur locked in CS and SiS does not exceed the solar elemental abundance of sulfur (i.e., $f_{0}(\mathrm{CS})+f_{0}(\mathrm{SiS}) \leq 2.6 \times 10^{-5}$; Asplund et al. 2009) in any envelope of our sample. Such a larger radial extent for CS, compared with $\mathrm{SiO}$ and $\mathrm{SiS}$, is consistent with the lower photodissociation rate calculated for CS compared to that computed for SiO (Heays et al. 2017; Pattillo et al. 2018; see Sect. 6.4) and with the larger emission size observed for $\mathrm{CS}$ with respect to $\mathrm{SiO}$ and SiS in IRC +10216 (Velilla Prieto et al. 2019).

In a recent study, Danilovich et al. (2018) derived empirical relations between $r_{e}$ and $\dot{M} / V_{\text {exp }}$ for SiS and CS from a limited sample of M-, C-, and S-type stars. We noticed that implementing their empirical relations in our model calculations resulted in anomalously high abundances for $\mathrm{CS}$ and $\mathrm{SiS}$ for some low mass loss rate envelopes, for example, for W Ori $f_{0}(\mathrm{CS})=3.2 \times 10^{-5}$ and $f_{0}(\mathrm{SiS})=8.8 \times 10^{-5}$, which imply a sulfur abundance well above the solar value. The main lesson from these calculations is that the empirical relations derived by Danilovich et al. (2018) cannot be safely extrapolated outside the relatively narrow range of $\dot{M} / V_{\text {exp }}$ over which they were derived. We therefore decided not to adopt the CS and SiS scaling laws of these authors. It is worth noting that Danilovich et al. (2018) derived larger $e$-folding radii for $\mathrm{CS}$ than for $\mathrm{SiS}$, which again points to $\mathrm{CS}$

\footnotetext{
4 Prasad \& Huntress (1980) reported a photodissociation rate of $1.0 \times 10^{-10} \mathrm{~s}^{-1}$ for SiS. Although it is difficult to trace the exact origin of this number, we suspect that it was most likely assumed to be equal to that of $\mathrm{SiO}$.
}

being more extended than SiS in agreement with the above arguments. Meanwhile, their SiS extent is smaller than that of $\mathrm{SiO}$ derived by González Delgado et al. (2003), which could point to $\mathrm{SiS}$ being photodissociated faster than $\mathrm{SiO}$.

In summary, to model the emission lines of $\mathrm{CS}, \mathrm{SiO}$, and SiS and determine their abundance in the observed sources, we constructed a model of the envelope for each source with the parameters given in Table 1 and adopting the abundance distribution given by Eq. (3), using Eqs. (4) and (5) accordingly, and performed excitation and radiative transfer calculations by varying the initial fractional abundance relative to $\mathrm{H}_{2}, f_{0}$ in Eq. (3), until the calculated line profiles matched the observed profiles. We chose the model that results in the best overall agreement between calculated and observed line profiles as the best-fit model. More specifically, our criterion was to match the line area of the calculated profile and the observed profile, and so we scaled the derived abundance until the line area is reproduced. In those cases where no line is detected, we derive upper limits to the corresponding molecular abundance by choosing the maximum abundance that results in line intensities compatible with the noise level of the observations.

\section{Results from line modeling}

The calculated line profiles resulting from our best-fit LVG model for each of the sources are shown in blue in Fig. 1 for CS, Fig. 2 for SiO, and Figs. 3 and 4 for SiS, where they are compared with the observed line profiles (black histograms). We note that the overall agreement of the model is good given that our criterion of matching the line areas is fulfilled as described in the previous section. Concerning the line shapes, globally, the agreement is good as well.To facilitate the differentiation between line detections and non-detections, the calculated line profiles of the latter are plotted in red. In the case of CS in U Cam, the contribution from the present-day wind, which is that we are interested in modeling, is shown in green.

In Fig. 5 we show some salient features of the excitation and radiative transfer calculations for two envelopes, STCam and CRL 190, which are representative of very different envelope densities. While ST Cam lies in the lower range, with $\dot{M} / V_{\exp }=$ $1.5 \times 10^{-8} M_{\odot} \mathrm{yr}^{-1} \mathrm{~km}^{-1} \mathrm{~s}, \mathrm{CRL} 190$ lies at the higher end, with $\dot{M} / V_{\text {exp }}=3.7 \times 10^{-6} M_{\odot} \mathrm{yr}^{-1} \mathrm{~km}^{-1}$ s. The bottom panels of Fig. 5 show the calculated ratio of excitation temperature to gas kinetic temperature $\left(T_{\mathrm{ex}} / T_{k}\right)$ for the observed lines of $\mathrm{CS}$ and $\mathrm{SiO}$ and that of the observed lines of $\mathrm{SiS}$ as a function of radius. We see that the rotational levels involved in the targeted transitions of the three molecules are thermalized $\left(T_{\mathrm{ex}} / T_{k}=1\right)$ in the hot and dense inner regions, while as the radial distance from the star increases and the gas density decreases, the rotational levels deviate from thermalization. Concretely, lines become increasingly suprathermally excited $\left(T_{\mathrm{ex}} / T_{k}>1\right)$ with increasing radius, an effect that is largely caused by IR pumping. Therefore, IR pumping plays an important role in the excitation of the rotational transitions of these molecules. The much lower mass loss rate of ST Cam compared to CRL 190 implies substantially lower densities in the envelope and thus in ST Cam rotational populations start to deviate from thermalization at shorter radii than in CRL 190. The fact that the observed lines are not thermalized throughout most of the envelope, especially at low mass loss rates, stresses the need for non-LTE excitation calculations. For the low-mass loss rate ST Cam, the models without IR pumping for $\mathrm{SiO}$ and $\mathrm{CS}$ display a peculiar behavior caused by the excitation temperature becoming negative from intermediate regions of the envelope. Including IR pumping makes this behavior 
ST Cam
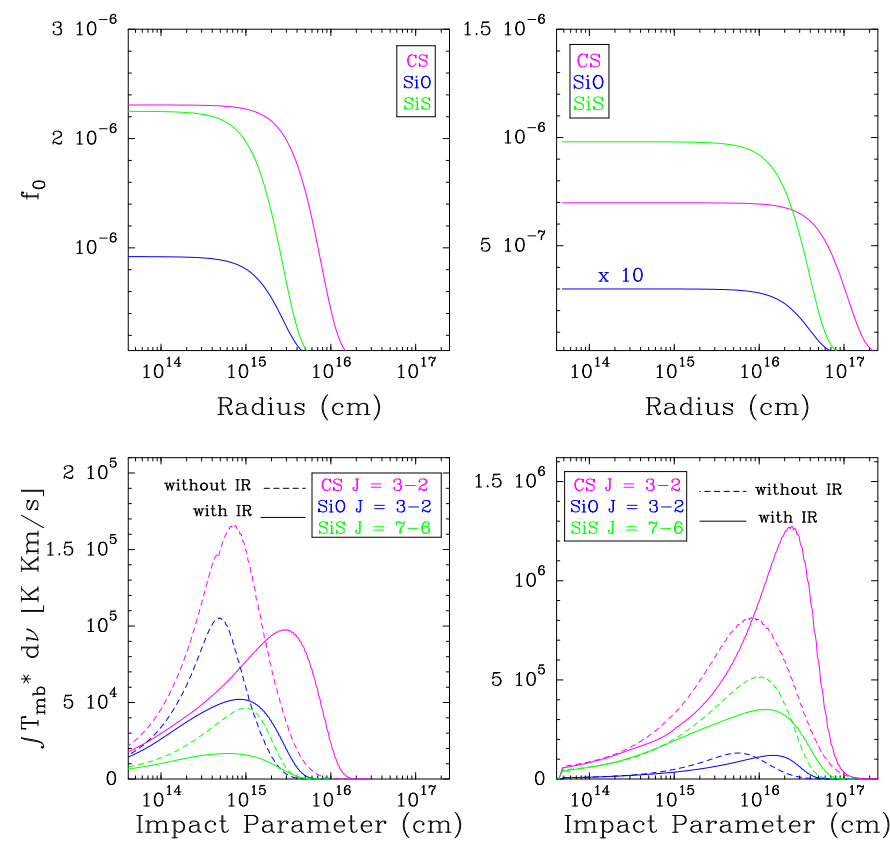

Impact Parameter $(\mathrm{cm})$
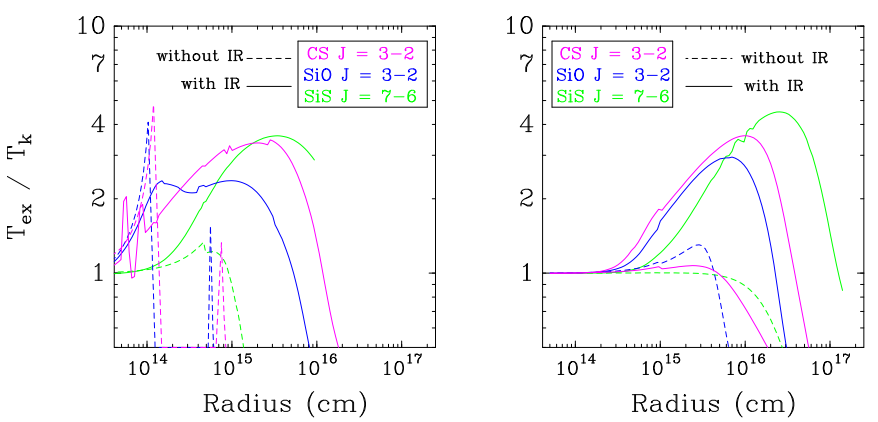

Fig. 5. Various features from the excitation and radiative transfer models for a low mass loss rate object (ST Cam) and a high mass loss rate object (CRL 190). Top panels: radial abundance profiles of CS, SiO, and SiS. Middle panels: calculated velocity-integrated intensities for the observed lines of $\mathrm{CS}, \mathrm{SiO}$, and $\mathrm{SiS}$ as functions of the impact parameter. Bottom panels: calculated ratio of excitation temperature to kinetic temperature $\left(T_{\mathrm{ex}} / T_{k}\right)$ as a function of radius for observed lines of CS, $\mathrm{SiO}$, and $\mathrm{SiS}$. Solid lines correspond to the model in which IR pumping is included, whereas dashed lines correspond to the model excluding IR pumping. The SiS abundance in ST Cam corresponds to an upper limit.

disappear. We note the SiS lines seem to end so abruptly because of the choice of the envelope outer edge in our model, where we chose the envelope to end at a radius at which the fractional abundance has dropped by a factor of $10^{5}$.

The middle panels of Fig. 5 show the velocity-integrated intensity of the observed lines as a function of the impact parameter (solid lines). We see that in ST Cam the maximum contribution to the line emission comes from regions at a few $10^{15} \mathrm{~cm}$ from the star, while in the case of CRL 190 the regions located at radii of a few $10^{16} \mathrm{~cm}$ contribute the most to the observed emission. Therefore, most of the emission of the observed lines arises from intermediate and outer regions of the envelope, rather than from inner regions. Moreover, the $\lambda 2 \mathrm{~mm}$ lines studied in this work probe regions where the abundance falloff has already started, which is especially marked in objects with low mass loss rates such as ST Cam (compare middle and top panels in Fig. 5). This fact explains why the observed lines are sensitive to both
Table 3. Fractional abundances of $\mathrm{CS}, \mathrm{SiO}$, and $\mathrm{SiS}$ derived.

\begin{tabular}{|c|c|c|c|c|c|}
\hline Name & $\begin{array}{c}\dot{M} \\
\left(M_{\odot} \mathrm{yr}^{-1}\right)\end{array}$ & $\begin{array}{c}V_{\exp } \\
\left(\mathrm{km} \mathrm{s}^{-1}\right)\end{array}$ & $f_{0}(\mathrm{CS})$ & $f_{0}(\mathrm{SiO})$ & $f_{0}(\mathrm{SiS})$ \\
\hline IRC + 10216 & $2.0 \times 10^{-5}$ & 14.5 & $1.1 \times 10^{-6}$ & $2.6 \times 10^{-7}$ & $1.3 \times 10^{-6}$ \\
\hline CIT 6 & $6.0 \times 10^{-6}$ & 17 & $6.4 \times 10^{-6}$ & $1.5 \times 10^{-6}$ & $4.8 \times 10^{-6}$ \\
\hline CRL 3068 & $2.5 \times 10^{-5}$ & 14.5 & $7.4 \times 10^{-7}$ & $1.4 \times 10^{-7}$ & $3.8 \times 10^{-6}$ \\
\hline S Cep & $1.2 \times 10^{-6}$ & 22.5 & $9.9 \times 10^{-6}$ & $9.4 \times 10^{-6}$ & $1.9 \times 10^{-6}$ \\
\hline IRC +30374 & $1.0 \times 10^{-5}$ & 25 & $1.0 \times 10^{-5}$ & $5.0 \times 10^{-6}$ & $7.2 \times 10^{-6}$ \\
\hline YCVn & $1.5 \times 10^{-7}$ & 7 & $5.7 \times 10^{-6}$ & $8.1 \times 10^{-7}$ & $<8.0 \times 10^{-7}$ \\
\hline LP And & $7.0 \times 10^{-6}$ & 14.5 & $3.6 \times 10^{-6}$ & $1.0 \times 10^{-6}$ & $7.0 \times 10^{-6}$ \\
\hline V Cyg & $1.6 \times 10^{-6}$ & 12 & $3.3 \times 10^{-6}$ & $2.9 \times 10^{-6}$ & $4.0 \times 10^{-6}$ \\
\hline UU Aur & $2.4 \times 10^{-7}$ & 10.6 & $3.0 \times 10^{-7}$ & $2.5 \times 10^{-7}$ & $<6.1 \times 10^{-7}$ \\
\hline V384 Per & $2.3 \times 10^{-6}$ & 15.5 & $8.4 \times 10^{-6}$ & $6.4 \times 10^{-6}$ & $1.0 \times 10^{-5}$ \\
\hline IRC +60144 & $3.7 \times 10^{-6}$ & 19.5 & $7.3 \times 10^{-6}$ & $9.5 \times 10^{-6}$ & $1.0 \times 10^{-5}$ \\
\hline $\mathrm{UCam}^{(a)}$ & $2.0 \times 10^{-7}$ & 13 & $1.9 \times 10^{-5}$ & $1.0 \times 10^{-5}$ & $<4.1 \times 10^{-6}$ \\
\hline V636 Mon & $5.8 \times 10^{-6}$ & 20 & $2.0 \times 10^{-6}$ & $1.7 \times 10^{-6}$ & $9.6 \times 10^{-7}$ \\
\hline IRC +20370 & $3.0 \times 10^{-6}$ & 14 & & & \\
\hline R Lep & $8.7 \times 10^{-7}$ & 17.5 & $3.6 \times 10^{-6}$ & $5.7 \times 10^{-6}$ & $<1.1 \times 10^{-6}$ \\
\hline W Ori & $7.0 \times 10^{-8}$ & 11 & $2.1 \times 10^{-5}$ & $3.6 \times 10^{-6}$ & $<4.9 \times 10^{-6}$ \\
\hline CRL 67 & $1.1 \times 10^{-5}$ & 16 & $3.5 \times 10^{-6}$ & $1.1 \times 10^{-6}$ & $4.6 \times 10^{-6}$ \\
\hline CRL 190 & $6.4 \times 10^{-5}$ & 17 & $7.0 \times 10^{-7}$ & $3.0 \times 10^{-8}$ & $9.8 \times 10^{-7}$ \\
\hline S Aur & $4.0 \times 10^{-7}$ & 24.5 & $9.3 \times 10^{-6}$ & $6.9 \times 10^{-6}$ & $<3.3 \times 10^{-6}$ \\
\hline V Aql & $1.4 \times 10^{-7}$ & 8 & $1.0 \times 10^{-5}$ & $2.5 \times 10^{-6}$ & $<2.3 \times 10^{-6}$ \\
\hline CRL 2513 & $2.0 \times 10^{-5}$ & 25.5 & $3.1 \times 10^{-6}$ & $1.3 \times 10^{-6}$ & $2.8 \times 10^{-6}$ \\
\hline CRL 2477 & $1.1 \times 10^{-4}$ & 20 & $2.7 \times 10^{-7}$ & $1.0 \times 10^{-7}$ & $1.7 \times 10^{-6}$ \\
\hline CRL 2494 & $7.5 \times 10^{-6}$ & 20 & $7.0 \times 10^{-6}$ & $2.9 \times 10^{-6}$ & $1.1 \times 10^{-5}$ \\
\hline RV Aqr & $2.3 \times 10^{-6}$ & 15 & $7.7 \times 10^{-6}$ & $5.5 \times 10^{-6}$ & $4.6 \times 10^{-6}$ \\
\hline ST Cam & $1.3 \times 10^{-7}$ & 8.9 & $2.3 \times 10^{-6}$ & $<9.2 \times 10^{-7}$ & $<2.2 \times 10^{-6}$ \\
\hline
\end{tabular}

Notes. ${ }^{(a)} \mathrm{U}$ Cam has a present-day wind and a detached shell expanding away from the central star (for further details see Schöier et al. 2005). The values of $f_{0}$ given in this table correspond to the present-day wind.

the fractional abundance and the radial extent, and why adopting a more compact (extended) distribution would require a higher (lower) fractional abundance to reproduce the observed lines.

Infrared pumping not only plays an important role in regulating the excitation of the observed lines but also in determining their emission distribution. The bottom panels in Fig. 5 show that the emission is more compact if IR pumping is not included (dashed lines) than if it is taken into account (solid lines). Therefore, IR pumping results in a more extended emission distribution with an impact on the emerging line intensity. In fact, neglecting IR pumping results in a systematic decrease in the integrated line intensities of $\mathrm{CS} J=3-2$ and $\mathrm{SiO} J=3-2$ of $\sim 60$ and $\sim 50 \%$, respectively, on average. In the case of SiS $J=7-6$ and $J=8-7$ the effect of excluding IR pumping is not as systematic as with $\mathrm{CS}$ and $\mathrm{SiO}$ as it leads to a decrease of the line intensities in some sources and an increase in other sources. If higher $J$ lines of SiS were targeted (above $J=10-9$ ) the effect of excluding IR pumping would be a systematic decrease in the integrated line intensities.

\section{Discussion}

\subsection{Fractional abundances}

The abundances derived for $\mathrm{CS}, \mathrm{SiO}$, and $\mathrm{SiS}$ in the 25 carbonrich envelopes studied are summarized in Table 3. For some of these sources, abundances have been previously reported. For example, in IRC +10216 the abundances of CS, SiO, and SiS were derived by Agúndez et al. (2012) from a multiline study including lines from vibrationally excited states. These authors found abundances relative to $\mathrm{H}_{2}$ of $7 \times 10^{-7}$ for $\mathrm{CS}$, $1.8 \times 10^{-7}$ for $\mathrm{SiO}$, and $1.3 \times 10^{-6}$ for $\mathrm{SiS}$, which are in very good agreement with the abundances derived in this work. 

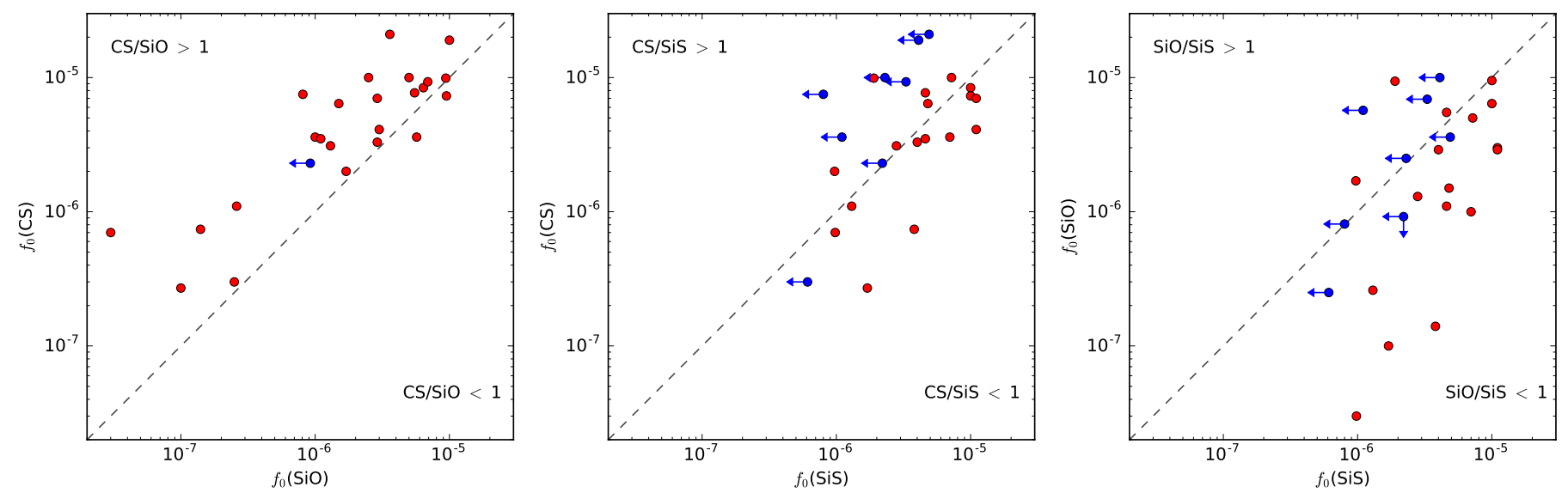

Fig. 6. Comparison of abundances between different pairs of molecules. The plots show the derived fractional abundances relative to $\mathrm{H}_{2}$ of CS vs. SiO (left panel), CS vs. SiS (middle panel), and SiO vs. SiS (right panel). Those sources with non-detections are denoted with blue arrows. Diagonal dashed lines indicate where the abundances of the two molecules become equal.

Olofsson et al. (1993) looked for CS $J=2-1$ emission in a large sample of carbon stars, 12 of which are in our sample. However, in about half of these stars the line was not detected and only loose abundance upper limits could be derived. These authors used a relatively simple method for estimating the molecular abundances, which is based on an analytical expression and where they assumed a constant excitation temperature for simplicity. In those sources where CS was detected, the abundances derived were very high, often greater than the maximum amount obtainable for solar abundance of sulfur. They suggested that these anomalously high abundances probably originate from uncertainties in the envelope model. In another study, Bujarrabal et al. (1994) surveyed a large sample of evolved stars in lines of several molecules including CS, SiO, and SiS. Their sample contains 16 C-rich AGB stars, 12 of which are also in our sample. These authors derived the abundances using an analytical expression that estimated only a lower limit if the line was optically thick. In general, their $\mathrm{CS}, \mathrm{SiO}$, and $\mathrm{SiS}$ abundances are lower than ours by a factor of a few and not higher than a factor of 10, apart from U Cam, where our derived CS and $\mathrm{SiO}$ abundances are one and two orders of magnitude, respectively, higher than theirs. Bujarrabal et al. (1994) remarked that their abundances may be underestimated owing to optical depth effects.

More recently, Schöier et al. (2006b) surveyed a sample of 19 C-rich AGB stars and detected $\mathrm{SiO}$ line emission in all of these objects. They performed radiative transfer calculations to derive abundances adopting, similar to us, an abundance distribution based on the scaling law established by González Delgado et al. (2003) for $\mathrm{SiO}$ in M-type stars. We share 13 sources with the former. Our $\mathrm{SiO}$ abundances are in good agreement with theirs for some sources, while for others there are significant differences. In LP And, for example, our derived $\mathrm{SiO}$ abundance is higher than theirs by almost a factor of ten, but on the other hand, our derived value in UU Aur is one order of magnitude lower than theirs, probably owing to differences in both the observations and the model. Later on, Schöier et al. (2007) reported on the detection of SiS line emission in 14 carbon stars, most of which are included in our sample. In general, the SiS abundances derived by us are similar to theirs, except for LP And, where we derive a SiS abundance higher than theirs by a factor of $\sim 6$.

In this work, the fractional abundances relative to $\mathrm{H}_{2}$ derived range between $2.7 \times 10^{-7}$ and $2.1 \times 10^{-5}$ for $\mathrm{CS}, 3 \times 10^{-8}$ and
$1 \times 10^{-5}$ for $\mathrm{SiO}$, and $<6.1 \times 10^{-7}$ and $1.1 \times 10^{-5}$ for $\mathrm{SiS}$. Silicon monoxide is the molecule experiencing the largest variations (more than two decades) from source to source, while CS abundances span over two decades, and SiS shows the most uniform abundances across sources (with variations of less than two decades). We find that CS is systematically more abundant than $\mathrm{SiO}$ in most of the sources (see left panel of Fig. 6, where it is seen that most sources fall in the region of $\mathrm{CS} / \mathrm{SiO}>1$ ). We also note that $\mathrm{SiO}$ tends to be less abundant than $\mathrm{SiS}$; the right panel of Fig. 6 shows that for most sources $\mathrm{SiO} / \mathrm{SiS} \lesssim 1$, while only in a few $\mathrm{SiO} / \mathrm{SiS}$ is greater than one. Therefore, $\mathrm{SiS}$ seems to be a more efficient gas-phase reservoir of silicon than $\mathrm{SiO}$ in most carbon star envelopes. When comparing between CS and $\mathrm{SiS}$, the two major gas-phase molecular reservoirs of sulfur in C-rich envelopes (see, Danilovich et al. 2018 and, e.g., the case of IRC+10216; Agúndez et al. 2012), we notice that in some sources such as IRC +30374 and U Cam, CS is more abundant than SiS, while in others (e.g., CRL 3068 and CRL 2494) the contrary is found. Overall, the data points in the middle panel of Fig. 6 fall along the line defined by $f_{0}(\mathrm{CS})=f_{0}(\mathrm{SiS})$, with no clear preference for either the $\mathrm{CS} / \mathrm{SiS}>1$ or the $\mathrm{CS} / \mathrm{SiS}<1$ sides.

We can therefore extract as statistically meaningful conclusions that in carbon star envelopes CS and SiS have abundances of the same order, and that $\mathrm{SiO}$ is in general less abundant than $\mathrm{CS}$ and SiS. This is most likely a chemical feature related to C-rich AGB envelopes. Danilovich et al. (2018) determined the $\mathrm{CS}$ and SiS abundances in a sample of AGB stars, and likewise found comparable CS and SiS abundances in C-rich AGB stars, while in their O-rich sample, SiS was found to be systematically higher than CS. S stars were also found to have similar abundances. In this line, it is interesting to see what TE predicts for these molecules in C-rich AGB atmospheres. In Fig. 7 we show the calculated fractional abundances of $\mathrm{CS}, \mathrm{SiO}$, and $\mathrm{SiS}$ as a function of radial distance from the star for various mass loss rates. The underlying physical scenario adopted for the TE calculations is the same as adopted in Massalkhi et al. (2018), where we used the radial profiles of density and temperature of IRC +10216 (Agúndez et al. 2012; see downward revision on the density profile by Cernicharo et al. 2013) and scaled the density profile up or down depending on the mass loss rate, that is in the range of $10^{-7}-10^{-4} M_{\odot} \mathrm{yr}^{-1}$. Therefore, the TE calculations adopt different density profiles to account for low and 

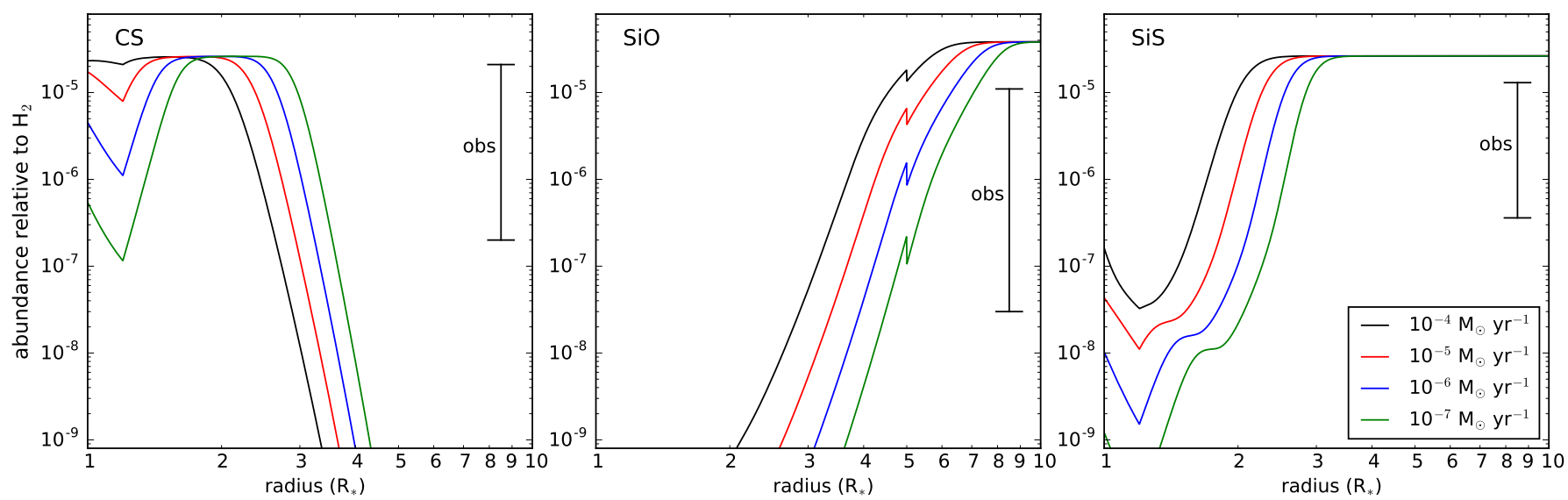

Fig. 7. Calculated fractional abundances of CS (left panel), SiO (middle panel), and SiS (right panel) under TE as a function of distance to the AGB star for various mass loss rates. The range of observed abundances is indicated.

high mass loss rates. We see that CS is predicted to have a high abundance (locking most of the available sulfur) in the close surroundings of the AGB star (at 1-3 $R_{*}$ ), while at farther distances its TE abundance vanishes to very low levels. On the other hand, SiS has almost the opposite behavior, with low abundance levels close to the star and a very high abundance beyond $2-3 R_{*}$. The fact that CS and SiS are observed with similar abundances in our sample of envelopes is consistent with the abundances of these molecules being quenched to their TE values at radii of 2-3 $R_{*}$, which agrees with findings from the study of IRC +10216 (Agúndez \& Cernicharo 2006; Agúndez et al. 2012). The process of abundance quenching is expected because as the gas expands the temperature and density drop and chemical reactions become slower and eventually too slow to proceed efficiently so that abundances are not further modified. Silicon monoxide shows a calculated radial TE abundance profile similar to that of $\mathrm{SiS}$ but shifted to larger radii. That is, $\mathrm{SiO}$ has a vanishingly small TE abundance close to the star, although calculations predict that this will reach a very high abundance (trapping most of the available silicon) beyond 5-8 $R_{*}$. The fact that the TE abundance of $\mathrm{SiO}$ is only high at a relatively large distance from the star is probably at the origin of the lower observed abundance of $\mathrm{SiO}$ compared to $\mathrm{CS}$ and $\mathrm{SiS}$. In any case, given the range of $\mathrm{SiO}$ abundances derived from observations, $\mathrm{SiO}$ must quench its abundance to the TE value at somewhat larger radii than for CS and SiS. Predictions from a chemical kinetics model of the inner wind of AGB stars, including shocks driven by the pulsation of the star (Cherchneff 2006), indicate that for a C-rich object with a $\mathrm{C} / \mathrm{O}$ elemental abundance ratio of 1.1, the abundances of $\mathrm{CS}$, $\mathrm{SiO}$, and $\mathrm{SiS}$ injected into the expanding envelope would be similar within a factor of $\sim 1.5$, where CS is slightly more abundant than $\mathrm{SiO}$ and $\mathrm{SiS}$. Given the scatter in the relative abundances of $\mathrm{CS}, \mathrm{SiO}$, and $\mathrm{SiS}$ derived by us and the very concrete physical scenario of AGB wind adopted by Cherchneff (2006), it is difficult to establish meaningful conclusions regarding whether the relative abundances we derive may be ultimately driven by shock-induced chemistry in the inner wind.

It is interesting to note that regardless of which pair of molecules is chosen, the plots in Fig. 6 show a trend in which the higher the abundance of one molecule the more abundant the other is. That is, the abundances of $\mathrm{CS}, \mathrm{SiO}$, and $\mathrm{SiS}$ seem to scale with each other, so that there are envelopes in which the three molecules are abundant, while in others the three molecules maintain low abundance levels. This conclusion seems robust when considering $\mathrm{CS}$ and $\mathrm{SiO}$, although it may not be completely true concerning SiS because in some of the sources where $\mathrm{SiS}$ is not detected, SiS may have a low abundance while $\mathrm{CS}$ and $\mathrm{SiO}$ do not. This point is discussed in more detail below. We note that a correlation of this type was found by Danilovich et al. (2018) for CS and SiS in a sample including C-, M-, and S-type stars, although in that study the trend is considered tentative because of the small number of sources included.

In Fig. 8 we plot the fractional abundances $f_{0}$ derived for $\mathrm{CS}, \mathrm{SiO}$, and $\mathrm{SiS}$ as a function of the density in the envelope, evaluated through the quantity $\dot{M} / V_{\text {exp. }}$ In the case of CS, the data strongly suggest a negative correlation between CS abundance and envelope density. The same behavior is even more evident for $\mathrm{SiO}$. That is, as the density in the envelope increases the abundances of $\mathrm{CS}$ and $\mathrm{SiO}$ decrease. This behavior was already found for $\mathrm{SiO}$ in both M-type stars by González Delgado et al. (2003) and carbon stars by Schöier et al. (2006b) and was interpreted as an evidence of enhanced $\mathrm{SiO}$ adsorption onto dust grains (and thus depletion from the gas phase) with increasing density. Using a larger sample of carbon stars, we thus confirm the result found by Schöier et al. (2006b) for SiO. The same trend of decreasing abundance with increasing density was also found recently for $\mathrm{SiC}_{2}$, pointing to this molecule as a gas-phase precursor of silicon carbide dust around carbon stars (Massalkhi et al. 2018). We note that in the recent study by Danilovich et al. (2018), the abundances of CS derived in C-type AGB envelopes do not show the anticorrelation with envelope density found by us. The reason is that their sample of carbon stars, with just seven objects, is small and the range of $\dot{M} / V_{\text {exp }}$ covered does not include high density envelopes, of which those in our sample give better evidence of the trend of decreasing abundance with increasing density.

It is worth looking at the predictions of TE to investigate whether the anticorrelation between abundance and envelope density observed for $\mathrm{CS}$ and $\mathrm{SiO}$ could be caused by an effect of the density on the TE abundance of these molecules. As shown in Fig. 7, the main effect of an increase in the mass loss rate, and thus on the envelope density, on the abundances of CS and $\mathrm{SiO}$ is that the curves shift slightly to inner radii. If the radius at which the CS abundance is quenched to its TE value is the same for different mass loss rates, then the quenched abundance of CS would be lower for higher mass loss rates, in agreement with the observed behavior. It is however unlikely that the radius of abundance quenching is the same for different mass loss rates because higher densities would make the region of abundance quenching occur at larger radii. The reason is that higher densities 

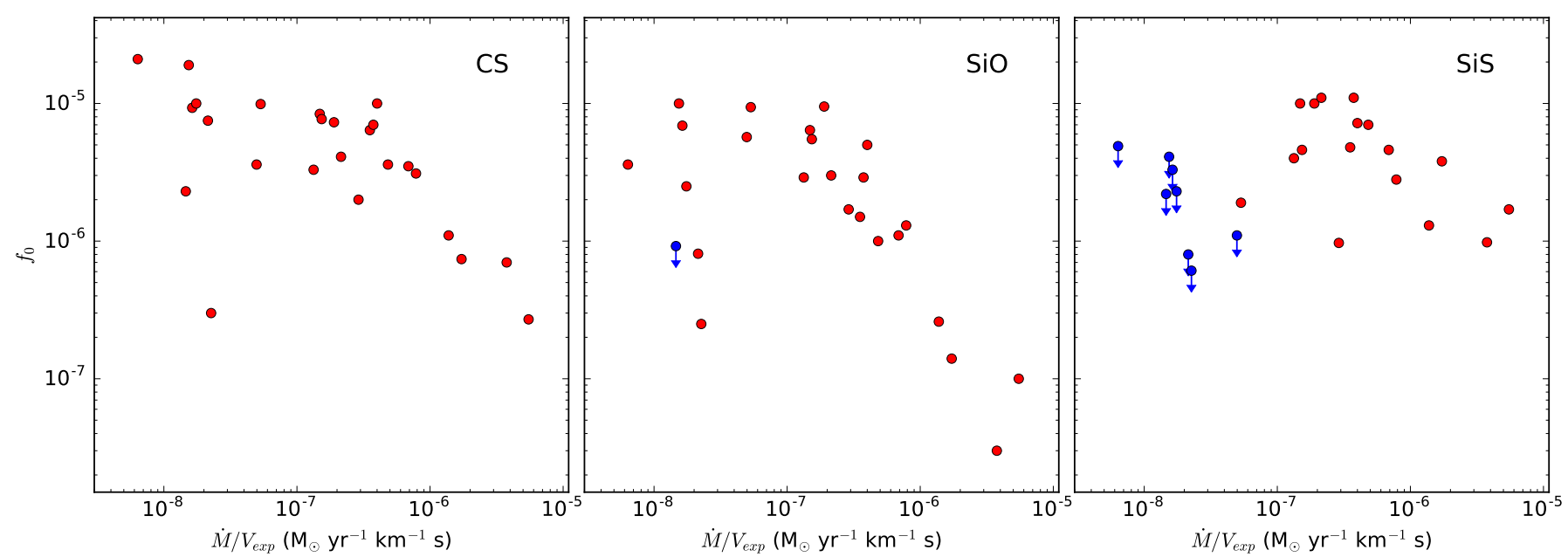

Fig. 8. Fractional abundances $f_{0}$ derived for CS (left panel), $\mathrm{SiO}$ (middle panel), and SiS (right panel) are plotted as a function of the envelope density proxy $\dot{M} / V_{\text {exp }}$ for the 25 C-rich envelopes studied here. Blue downward arrows represent upper limits to $f_{0}$.

imply shorter chemical timescales and a larger region in which TE prevails. This would result in an even more pronounced decrease of the quenched CS abundance with increasing density. Although qualitatively this scenario would be in agreement with the observed trend of decreasing abundance of CS with increasing density, a detailed chemical kinetics model is needed to obtain quantitative estimates. In the case of $\mathrm{SiO}$, a similar reasoning implies that the quenched abundance would be higher for higher densities (see Agúndez \& Cernicharo 2006) contrary to what observations indicate. It is therefore very unlikely that the observed decrease in the abundance of $\mathrm{SiO}$ with increasing density is caused by gas-phase chemistry. The most likely explanation is that $\mathrm{SiO}$ incorporates into dust grains and depletes from the gas phase with increasing density in the envelope.

The case of SiS deserves special attention. It is remarkable that $\mathrm{SiS}$ is not detected in envelopes with low mass loss rates below $10^{-6} M_{\odot} \mathrm{yr}^{-1}$, while it is detected in all sources with mass loss rates above that threshold. This fact, which has been noticed to some extent in previous observational studies (Bujarrabal et al. 1994; Schöier et al. 2007; Danilovich et al. 2018), is shown in this work in a robust way (see Table 3 and right panel of Fig. 8). The reason behind this behavior is not clear. The non-detection of $\mathrm{SiS}$ in these envelopes might either be due to a lack of sensitivity or a low fractional abundance of the molecule. Looking at the predictions of TE (right panel in Fig. 7), we note that for low mass loss rates the predicted abundance of $\mathrm{SiS}$ is indeed lower. This fact is even more marked if we take into account that the radius at which the abundance quenches to the TE value is expected to be shifted to inner radii for lower mass loss rates. A scenario of TE chemistry plus abundance quenching would be in agreement with objects with low mass loss rates having lower SiS abundances. If that is the underlying cause, it is however strange that the observed SiS abundances do not show a smooth variation with density but an abrupt differentiation between sources with and without $\mathrm{SiS}$ detection at $10^{-6} M_{\odot} \mathrm{yr}^{-1}$, and show no evidence of increasing SiS abundance with increasing density for those sources where SiS is detected. We suspect that the lack of SiS detections in objects with low mass loss rates could be caused by a lack of its constituent elements, which would be largely trapped in $\mathrm{SiO}$ and $\mathrm{SiC}_{2}$ (in the case of silicon) and CS (regarding sulfur). As shown in this work and in Massalkhi et al. (2018), the molecules $\mathrm{SiO}, \mathrm{CS}$, and $\mathrm{SiC}_{2}$ (probably also $\mathrm{Si}_{2} \mathrm{C}$; Cernicharo et al. 2015) become very abundant in C-rich objects with low mass loss rates. This suspicion however would need to be corroborated with a detailed chemical kinetics model of the inner wind of envelopes with different mass loss rates. Apart from the upper limits to the abundance of SiS in objects with low mass loss rates, the SiS abundances derived through positive detections show hints of decreasing $\mathrm{SiS}$ abundance with increasing density (right panel in Fig. 8). This has to be seen as tentative, however, and in any case it is not as evident as in the cases of CS and $\mathrm{SiO}$. Therefore, if the tentative decrease in the abundance of SiS with increasing envelope density is interpreted in terms of adsorption onto dust grains, we can conclude that $\mathrm{SiS}$ is not incorporated into dust grains to an extent as important as that of $\mathrm{SiO}$.

Schöier et al. (2007) found that the SiS abundance does not show any particular correlation with the envelope density for C-rich envelopes. However, they found a slightly better fit to their observations for the case of IRC +10216 by including a compact SiS component with a fractional abundance $1.7 \times 10^{-5}$ out to a radius of $5 \times 10^{14} \mathrm{~cm}$, which could imply an SiS abundance gradient in line with the results found by Agúndez et al. (2012). Introducing an inner high-abundance SiS component also produced a better fit for the oxygen-rich IK Tau implying SiS depletion. This result is similar to that found by Decin et al. (2010b) when modeling low- and high-excitation lines, however a recent study by Danilovich et al. (2019), using sensitive ALMA observations to determine the SiS distribution in the envelope of IK Tau, does not reveal a compact inner region as previously found. Regardless, we do maintain that the evidence of SiS depletion in C-rich envelopes is weak.

In any case, in this work we investigated $\mathrm{SiS}$ in a larger sample of carbon stars covering a broader range of envelope densities than previously studied, which permitted us to clearly see a systematic non-detection of $\mathrm{SiS}$ at low mass loss rates and a tentative negative correlation between $\mathrm{SiS}$ abundance and envelope density seen at high mass loss rates.

\subsection{MgS dust: possible gas-phase precursors}

A common characteristic that is seen in C-rich evolved stars is the presence of a prominent IR emission band that is centered around $30 \mu \mathrm{m}$. The feature was discovered by Forrest et al. (1981) in dusty carbon-rich environments; Goebel \& Moseley (1985) first suggested that magnesium sulfide (MgS) dust may 

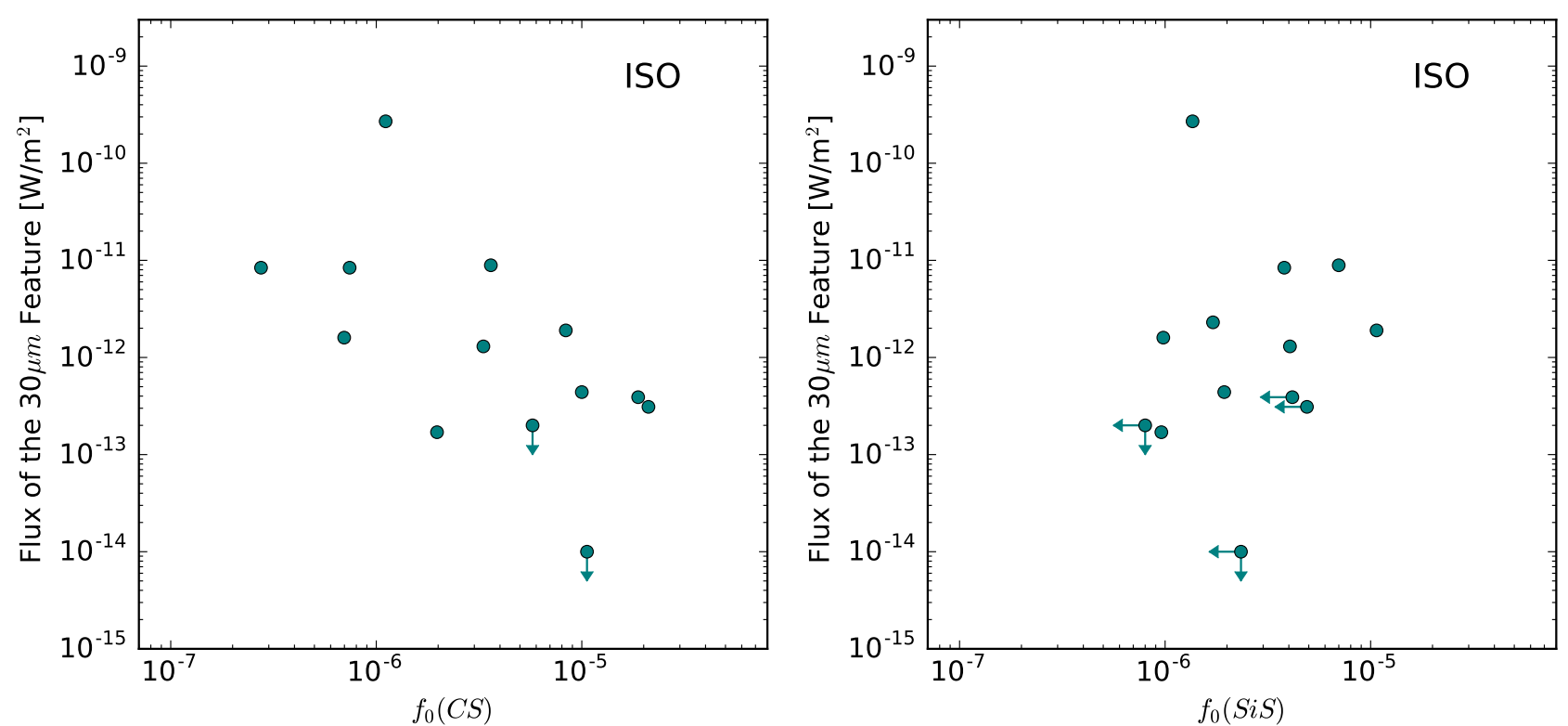

Fig. 9. Flux of the MgS dust feature at $30 \mu \mathrm{m}$ observed by ISO (Hony et al. 2002) vs. the fractional abundance of CS (left panel) and SiS (right panel) derived in this work.

be responsible for this spectral feature and this suggestion has been widely accepted since then. However, the carrier is still argued upon. One of the major concerns is regarding the sulfur abundance that is required to explain the observed emission. Zhang et al. (2009) argued that the amount of $\mathrm{MgS}$ required to explain the power emitted by the $30 \mu \mathrm{m}$ feature in the post-AGB, HD 56126, would require ten times more atomic sulfur than available in the ejected envelope. Zhukovska \& Gail (2008) found the only way MgS dust can form for C-rich AGB stars is by precipitation on preexisting silicon carbide grains. Lombaert et al. (2012) then discussed that if the grains were of a heterogeneous composite nature, meaning if $\mathrm{MgS}$ dust forms in a layer coated around an amorphous carbon/SiC core grain, then there would not be an abundance constraint. Investigating the formation mechanism of $\mathrm{MgS}$ dust is out of the scope of this paper. However, we can investigate if there is a connection between the sulfur bearing molecules studied in this work and the $30 \mu \mathrm{m}$ feature attributed to $\mathrm{MgS}$ dust. This way we could identify if any of these S-bearing molecules could act as precursors of $\mathrm{MgS}$ dust in the ejecta of AGB stars. More specifically, we aim to investigate whether there is a relation between the derived fractional abundances of $\mathrm{CS}$ and $\mathrm{SiS}$ and the strength of the $30 \mu \mathrm{m}$ feature.

Hony et al. (2002) carried out an observational study of the $30 \mu \mathrm{m}$ feature of a large number of C-rich sources observed with the Infrared Space Observatory (ISO). There are 13 sources in common between their sample and ours. In the left panel of Fig. 9 we plot the flux of MgS dust versus the fractional abundance of CS for these 13 sources. We clearly see a trend in which the flux of $\mathrm{MgS}$ dust increases as the gas-phase abundance of CS decreases. If the flux of the $30 \mu \mathrm{m}$ feature is a good proxy of the amount of $\mathrm{MgS}$ dust and if the hypothesis that $\mathrm{CS}$ is a gas-phase precursor of dust in C-rich AGB stars is correct, then this result supports the idea that the decline in the abundance of CS with increasing envelope density is caused by a more efficient incorporation of CS on MgS dust. In the case of SiS there is no obvious correlation between the fractional abundance and the flux of the $30 \mu \mathrm{m}$ feature (see right panel of Fig. 9). The lack of correlation suggests that $\mathrm{SiS}$ does likely not play a role in the formation of $\mathrm{MgS}$ dust in these stars, although we note that the range of SiS abundances covered is limited. Smolders et al. (2012) carried out an observational study on a large sample of S-type stars and found that many stars that show the MgS emission feature also show emission peaks at 6.6 and $13.5 \mu \mathrm{m}$ due to molecular SiS. This fact led these authors to suggest that $\mathrm{MgS}$ dust could be formed as a consequence of a reaction between $\mathrm{Mg}$ and $\mathrm{SiS}$ in S-type stars in contrast with our conclusion that $\mathrm{CS}$, rather than $\mathrm{SiS}$, is a precursor of $\mathrm{MgS}$ dust in C-rich AGB stars.

\subsection{SiO and SiS as possible precursors of SiC dust}

Since both $\mathrm{SiO}$ and $\mathrm{SiS}$ are important $\mathrm{Si}$-carriers (Olofsson et al. 1982; Lucas et al. 1995; Agúndez et al. 2012), in this section we assess if there could be a relation between these molecules and the formation of SiC dust around AGB stars. We collected information on the Infrared Astronomical Satellite (IRAS) and the ISO data for the sources in our sample that exhibit the SiC dust emission feature at $11.3 \mu \mathrm{m}$. Sloan et al. (1998) analyzed the IRAS spectra of carbon stars with which we share 15 sources, and Yang et al. (2004) studied the ISO spectra of C-rich AGB stars with 9 sources in common with our sample. These authors determined the relative flux of $\mathrm{SiC}$ dust as the ratio of the integrated flux of the $11.3 \mu \mathrm{m}$ emission feature (after continuum substraction) divided by the integrated flux of the continuum. In Fig. 10 we plot the relative integrated flux of $\mathrm{SiC}$ dust versus the fractional abundance of $\mathrm{SiO}$ (left) and $\mathrm{SiS}$ (right) for the sources in our sample that have IRAS or ISO data. With the same reasoning as in the previous section, if the relative flux of $\mathrm{SiC}$ dust is a direct indicator of the amount of silicon carbide dust and the hypothesis that $\mathrm{SiO}$ and/or $\mathrm{SiS}$ contribute to the formation of $\mathrm{SiC}$ dust is correct, we would expect to see a trend in which the relative flux of $\mathrm{SiC}$ dust increases as the gas-phase abundance of $\mathrm{SiO}$ and/or SiS decreases. However, Fig. 10 does not show any clear trend indicating this; the same result was found between the gas-phase $\mathrm{SiC}_{2}$ and $\mathrm{SiC}$ dust by Massalkhi et al. (2018). Regardless, it is important to note that the relative flux of the $11.3 \mu \mathrm{m}$ $\mathrm{SiC}$ band is an observable quantity that may not be a direct indicator of the mass of silicon carbide dust in the envelope. The derivation of the amount of $\mathrm{SiC}$ dust in the envelope requires 

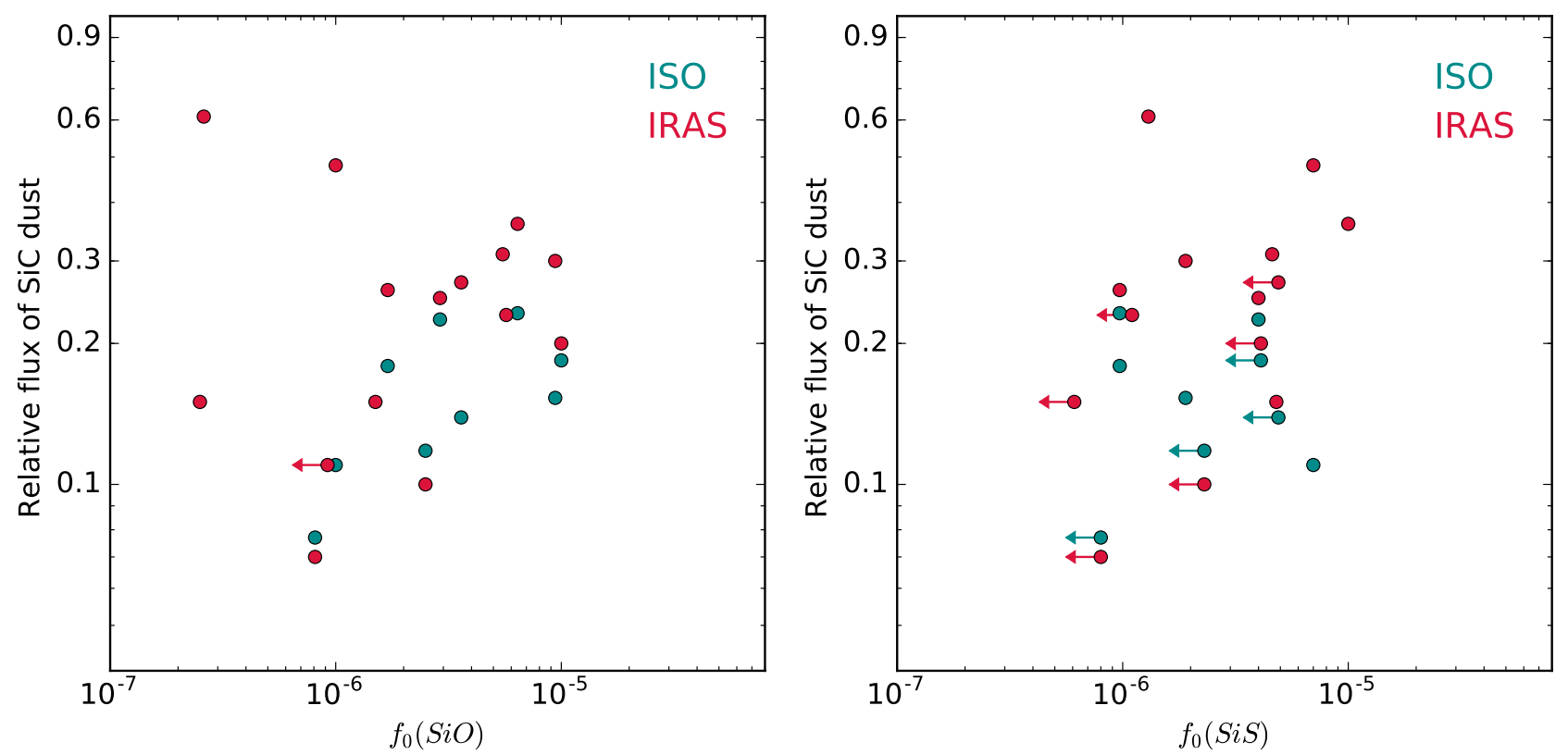

Fig. 10. Relative integrated flux of the $\mathrm{SiC}$ dust feature at $11.3 \mu \mathrm{m}$ taken from Sloan et al. (1998) and Yang et al. (2004) vs. the fractional abundance of $\mathrm{SiO}$ and $\mathrm{SiS}$ derived in this work.

a radiative transfer analysis that involves a meticulous description of the chemical composition, size, and temperature of dust throughout the envelope.

\subsection{Radial extent: photodissociation versus empirical relations}

As discussed in Sect. 4.2, the radial extent of $\mathrm{CS}, \mathrm{SiO}$, and $\mathrm{SiS}$ in carbon star envelopes is probably controlled by photodissociation from the ambient interstellar UV radiation field. However, given the aforementioned difficulties to account properly for the abundance falloff using photodissociation rates from the literature (in which case the radial extent is very likely underestimated), in this work we adopted a simple radial abundance distribution given by a Gaussian function with $e$-folding radii derived from empirical relations between $r_{e}$ and the wind density. These relations were ultimately based on a scaling law between $r_{e}$ and $\dot{M} / V_{\text {exp }}$ derived from a multiline study of $\mathrm{SiO}$ in M-type stars (González Delgado et al. 2003). In this work we adopted the same relation for $\mathrm{SiO}$, based on the assumption that $\mathrm{SiO}$ behaves similarly in envelopes around $\mathrm{M}$ - and C-type stars; for SiS we adopted the same relation, based on the assumption that $\mathrm{SiS}$ and $\mathrm{SiO}$ have similar emission sizes as mentioned previously; and for CS we adopted a modified version, based on arguments to avoid requiring a sulfur abundance higher than the solar abundance. If we consider as good the adopted empirical relations and we assume that the abundance falloff is entirely controlled by photodissociation, then it is possible to extract some useful conclusions about the photodissociation of these molecules. More specifically, we aim to find the unattenuated photodissociation rates that best reproduce the abundance falloff given by the adopted empirical laws for $r_{e}$.

The photodissociation model used for this exercise is the same employed in Massalkhi et al. (2018). Briefly, the radial variation of the fractional abundance can be expressed as (Jura \& Morris 1981; Huggins \& Glassgold 1982)

$\frac{\mathrm{d} f}{\mathrm{~d} r}=-\frac{\alpha}{V_{\exp }} \exp \left[-\left(\frac{r_{\mathrm{d}}}{r}\right)\right] f$,
Table 4. Photodissociation parameters.

\begin{tabular}{lccccc}
\hline \hline Molecule & $\alpha$ & $\beta$ & Ref. & $\alpha^{\prime}$ & $\langle$ Dev. $\rangle$ \\
\hline $\mathrm{CS}$ & $3.7 \times 10^{-10}$ & 2.32 & $\mathrm{a}$ & $1.5 \times 10^{-10}$ & $35 \%$ \\
$\mathrm{SiO}$ & $1.6 \times 10^{-9}$ & 2.66 & $\mathrm{~b}$ & $7 \times 10^{-10}$ & $31 \%$ \\
$\mathrm{SiS}$ & $1.6 \times 10^{-9}$ & 2.66 & $\mathrm{c}$ & $8 \times 10^{-10}$ & $47 \%$ \\
\hline
\end{tabular}

Notes. The values of $\alpha$ and $\beta$ are taken from the literature.

References. (a) Pattillo et al. (2018), (b) Heays et al. (2017), (c) assumed the same as $\mathrm{SiO}$. The parameter $\alpha^{\prime}$ are the values that best reproduce the empirical relations between $r_{e}$ and $\dot{M} / V_{\text {exp }}$ adopted in this study. The mean deviations $\langle$ Dev. $\rangle$ are also given (see text).

where $\alpha$ is the unattenuated photodissociation rate in $\mathrm{s}^{-1}$ and the photodissociation radius $r_{\mathrm{d}}$ is given by

$r_{\mathrm{d}}=\frac{\beta \dot{M}}{4 \pi V_{\mathrm{exp}} \bar{m}_{\mathrm{g}} 1.87 \times 10^{21}}$,

where $\beta$ is the dust shielding factor, $\bar{m}_{\mathrm{g}}$ is the average mass of gas particles in grams, and the numerical value is the canonical $N_{\mathrm{H}} / A_{\mathrm{V}}$ ratio for the local ISM (Bohlin et al. 1978). In Table 4 we list the unattenuated photodissociation rates $\alpha$ and dust shielding factors $\beta$ collected from the literature for $\mathrm{CS}, \mathrm{SiO}$, and $\mathrm{SiS}$, which were used as starting values. For each of the three molecules, we then varied the unattenuated photodissociation rate in small steps, computed the associated abundance radial profile for each envelope in the sample, and calculated the corresponding line profiles. To decide which photodissociation rate agrees best with the empirical abundance falloff, we computed for each envelope the difference between the velocity-integrated line intensity calculated with the photodissociation model and that calculated with the empirical falloff model and then estimated the mean difference.

The mean deviations found are plotted as a function of the photodissociation rate in Fig. 11. The optimal photodissociation rate $\alpha^{\prime}$ for which the lowest mean deviation is found is given in 


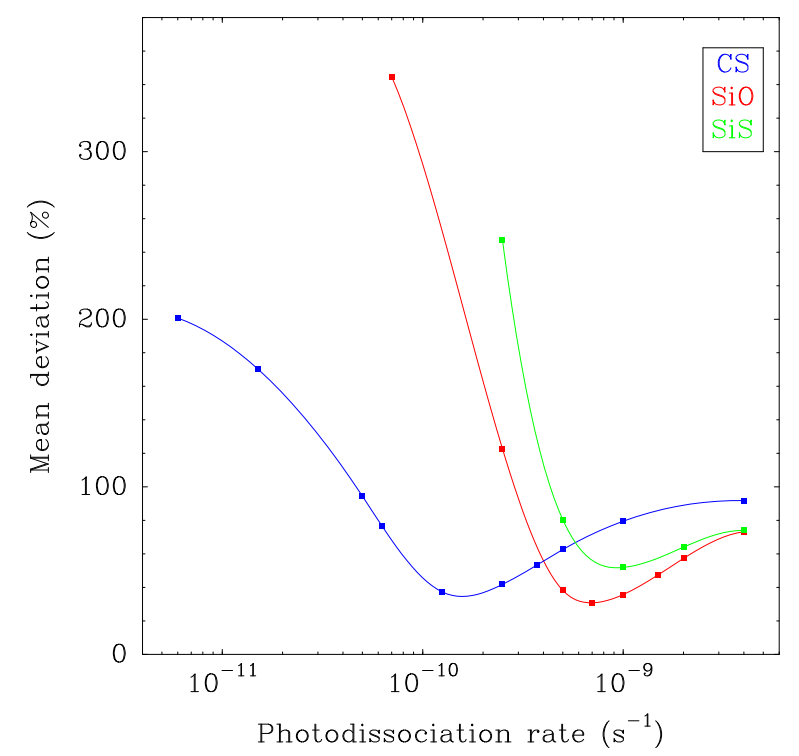

Fig. 11. The mean deviation between the calculated line areas with the photodissociation model and the model using empirical laws is plotted as a function of the unattenuated photodissociation rate adopted in the photodissociation model for $\mathrm{CS}, \mathrm{SiO}$, and $\mathrm{SiS}$.

the right side of Table 4 for each of the three molecules, together with the associated mean deviation. In the case of CS, we found that the lowest mean deviation (35\%) occurs for $\alpha^{\prime}=1.5 \times$ $10^{-10} \mathrm{~s}^{-1}$, while for $\mathrm{SiO}$ and $\mathrm{SiS}$ the lowest deviation (31 and $47 \%$, respectively) is found for photodissociation rates of $7 \times 10^{-10}$ and $8 \times 10^{-10} \mathrm{~s}^{-1}$, respectively. There are two interesting aspects to comment on. First, the optimal photodissociation rates found for $\mathrm{SiO}$ and $\mathrm{SiS}$ are very similar, while that of $\mathrm{CS}$ is significantly lower. These results merely reflect the adopted empirical abundance falloffs, which were the same for $\mathrm{SiO}$ and SiS, while for CS we adopted an empirical scaling law implying an outer abundance falloff. And second, the optimal photodissociation rates $\alpha^{\prime}$ are significantly lower than the literature values $\alpha$. This illustrates in a different way our initial suspicion that literature photodissociation rates underestimate the radial extent of $\mathrm{CS}, \mathrm{SiO}$, and $\mathrm{SiS}$. Whether, this finding implies that real photodissociation rates are indeed lower than the literature values or whether this points to a different $N_{\mathrm{H}} / A_{\mathrm{V}}$ ratio than the canonical interstellar value of Bohlin et al. (1978) is not yet clear.

\section{Conclusions}

In this work we used the IRAM $30 \mathrm{~m}$ telescope to survey a sample of 25 C-rich CSEs in the $J=3-2$ line of CS and SiO and in the $J=7-6$ and $J=8-7$ lines of SiS. We carried out excitation and radiative transfer calculations based on the LVG method to derive fractional abundances relative to $\mathrm{H}_{2}$. We found that the abundances of the three molecules are positively correlated with each other (especially those of $\mathrm{CS}$ and $\mathrm{SiO}$ ) and that while $\mathrm{CS}$ and $\mathrm{SiS}$ have similar abundances, $\mathrm{SiO}$ is present at a lower abundance level.

We also found a clear trend in which $\mathrm{SiO}$ and CS become less abundant as the envelope density increases. The depletion of CS with increasing density can be due to gas-phase chemistry in the inner wind or to incorporation onto dust grains. The latter scenario is favored by the fact that we find a negative correlation between the fractional abundance of CS and the $30 \mu \mathrm{m}$ feature attributed to $\mathrm{MgS}$ dust, which suggests that $\mathrm{CS}$ is a likely precursor of $\mathrm{MgS}$ dust grains in C-rich AGB envelopes. In the case of $\mathrm{SiO}$, the most likely explanation of the negative correlation between fractional abundance and envelope density is that $\mathrm{SiO}$ incorporates more efficiently onto dust grains at increasing density owing to the enhanced collision rate between particles and the acceleration of accretion and coagulation processes. Thus, both $\mathrm{CS}$ and $\mathrm{SiO}$ are probable candidates to act as gas-phase precursor of dust grains.

We find that SiS is systematically not detected in envelopes with mass loss rates below $10^{-6} M_{\odot} \mathrm{yr}^{-1}$, probably because of the increasing importance of other molecules that lock most silicon and sulfur ( $\mathrm{SiO}, \mathrm{SiC}_{2}$, and $\mathrm{CS}$ ) or because of a lack of sensitivity. The $\mathrm{SiS}$ abundances derived in the sources in which the molecule is detected suggest a tentative trend of decreasing abundance with increasing density. This trend indicates that $\mathrm{SiS}$ could also be incorporated into dust grains, although the nondetections clearly undermine this tentative trend. Nevertheless, this conclusion is not as robust as that of $\mathrm{CS}$ and $\mathrm{SiO}$.

Our conclusions on the role of $\mathrm{CS}, \mathrm{SiO}$, and $\mathrm{SiS}$ as gasphase precursors of dust are based on low energy lines, which probe post-condensation regions. More observations, in particular high-J lines and interferometric observations probing the inner regions of the envelopes, are needed to affirm the conclusions obtained in this study.

Acknowledgements. We are grateful to Otoniel Denis-Alpizar, Christian Balança, and Fabrice Dayou for providing the latest collisional rate coefficients of CS and $\mathrm{SiO}$. We thank the IRAM $30 \mathrm{~m}$ staff for their help during the observations. This research has made use of the SIMBAD database, operated at CDS, Strasbourg, France. We acknowledge funding support from the European Research Council (ERC Grant 610256: NANOCOSMOS) and from Spanish MINECO through grants AYA2012-32032 and AYA2016-75066-C2-1-P. M.A. thanks Spanish MINECO for funding support through the Ramón y Cajal programme (RyC-2014-16277).

\section{References}

Agúndez, M. 2009, PhD. Thesis, Universidad Autónoma de Madrid, Spain Agúndez, M., \& Cernicharo, J. 2006, ApJ, 650, 374

Agúndez, M., Fonfría, J. P., Cernicharo, J., et al. 2012, A\&A, 543, A48

Asplund, M., Grevesse, N., Sauval, A. J., \& Scott, P. 2009, ARA\&A, 47, 481

Balança, C., \& Dayou, F. 2017, MNRAS, 469, 1673

Bieging, J. H., \& Nguyen-Quang-Rieu. 1989, ApJ, 343, L25

Bohlin, R. C., Savage, B. D., \& Drake, J. F. 1978, ApJ, 224, 132

Boyle, R. J., Keady, J. J., Jennings, D. E., Hirsch, K. L., \& Wiedemann, G. R. 1994, ApJ, 420, 863

Bujarrabal, V., Fuente, A., \& Omont, A. 1994, A\&A, 285, 247

Castor, J. I. 1970, MNRAS, 149, 111

Cernicharo, J. 1985, The Infrared and Submillimetre Sky after COBE, eds. M. Signore \& C. Dupraz, IRAM Internal Rep., 52

Cernicharo, J., Guélin, M., \& Kahane, C. 2000, A\&AS, 142, 18

Cernicharo, J., Daniel, F., Castro-Carrizo, A., et al. 2013, ApJ, 778, L25

Cernicharo, J., McCarthy, M. C., Gottlieb, C. A., et al. 2015, ApJ, 806, L3

Chandra, S., Kegel, W. H., Le Roy, R. J., \& Hertenstein, T. 1995, A\&AS, 114, 175

Cherchneff, I. 2006, A\&A, 456, 1001

Danilovich, T., Ramstedt, S., Gobrecht, D., et al. 2018, A\&A, 617, A132

Danilovich, T., Richards, A. M. S., Karakas, A. I., et al. 2019, MNRAS, 484, 494

Dayou, F., \& Balança, C. 2006, A\&A, 459, 297

Decin, L., Cernicharo, J., Barlow, M. J., et al. 2010a, A\&A, 518, L143

Decin, L., De Beck, E., Brünken, S., et al. 2010b, A\&A, 516, A69

Denis-Alpizar, O., Stoecklin, T., Guilloteau, S., \& Dutrey, A. 2018, MNRAS, 478, 1811

Drira, I., Hure, J. M., Spielfiedel, A., Feautrier, N., \& Roueff, E. 1997, A\&A, 319,720

Forrest, W. J., Houck, J. R., \& McCarthy, J. F. 1981, ApJ, 248, 195

Gehrz, R. 1989, in Interstellar Dust, eds. L. J. Allamandola \& A. G. G. M Tielens, IAU Symp., Vol. 135, 445 
Goebel, J. H., \& Moseley, S. H. 1985, ApJ, 290, L35

González Delgado, D., Olofsson, H., Kerschbaum, F., et al. 2003, A\&A, 411, 123

Groenewegen, M. A. T., Sevenster, M., Spoon, H. W. W., \& Pérez, I. 2002, A\&A, 390,511

Heays, A. N., Bosman, A. D., \& van Dishoeck, E. F. 2017, A\&A, 602, A105

Herwig, F. 2005, ARA\&A, 43, 435

Hony, S., Waters, L. B. F. M., \& Tielens, A. G. G. M. 2002, A\&A, 390, 533

Huggins, P. J., \& Glassgold, A. E. 1982, ApJ, 252, 201

Jura, M., \& Morris, M. 1981, ApJ, 251, 181

Keady, J. J., \& Ridgway, S. T. 1993, ApJ, 406, 199

Kłos, J., \& Lique, F. 2008, MNRAS, 390, 239

Lique, F., \& Spielfiedel, A. 2007, A\&A, 462, 1179

Lombaert, R., de Vries, B. L., de Koter, A., et al. 2012, A\&A, 544, L18

Loup, C., Forveille, T., Omont, A., \& Paul, J. F. 1993, A\&AS, 99, 291

Lucas, R., Bujarrabal, V., Guilloteau, S., et al. 1992, A\&A, 262, 491

Lucas, R., Guélin, M., Kahane, C., Audinos, P., \& Cernicharo, J. 1995, Ap\&SS, 224, 293

Massalkhi, S., Agúndez, M., Cernicharo, J., et al. 2018, A\&A, 611, A29

Müller, H. S., Schlöder, F., Stutzki, J., \& Winnewisser, G. 2005, J. Mol. Struct., 742,215

Müller, H., McCarthy, M., Bizzocchi, L., et al. 2007, Phys. Chem. Chem. Phys., 9, 1579

Olofsson, H., Johansson, L. E. B., Hjalmarson, A., \& Nguyen-Quang-Rieu. 1982, A\&A, 107, 128

Olofsson, H., Carlstrom, U., Eriksson, K., Gustafsson, B., \& Willson, L. A. 1990 A\&A, 230, L13

Olofsson, H., Eriksson, K., Gustafsson, B., \& Carlstroem, U. 1993, ApJS, 87, 305

Pardo, J. R., Cernicharo, J., \& Serabyn, E. 2001, IEEE Trans. Antennas. Propag., 49,1683
Pattillo, R. J., Cieszewski, R., Stancil, P. C., et al. 2018, ApJ, 858, 10

Piñeiro, A. L., Tipping, R. H., \& Chackerian, C. 1987, J. Mol. Spectr., 125, 184

Prasad, S. S., \& Huntress, Jr. W. T. 1980, ApJS, 43, 1

Raymonda, J. W., Muenter, J. S., \& Klemperer, W. A. 1970, J. Chem. Phys., 52, 3458

Sanz, M. E., McCarthy, M. C., \& Thaddeus, P. 2003, J. Chem. Phys., 119, 11715

Schöier, F. L., \& Olofsson, H. 2001, A\&A, 368, 969

Schöier, F. L., Lindqvist, M., \& Olofsson, H. 2005, A\&A, 436, 633

Schöier, F. L., Fong, D., Olofsson, H., Zhang, Q., \& Patel, N. 2006a, ApJ, 649, 965

Schöier, F. L., Olofsson, H., \& Lundgren, A. A. 2006b, A\&A, 454, 247

Schöier, F. L., Bast, J., Olofsson, H., \& Lindqvist, M. 2007, A\&A, 473, 871

Schöier, F. L., Ramstedt, S., Olofsson, H., et al. 2013, A\&A, 550, A78

Sloan, G. C., Little-Marenin, I. R., \& Price, S. D. 1998, AJ, 115, 809

Smolders, K., Neyskens, P., Blommaert, J. A. D. L., et al. 2012, A\&A, 540, A72

Sobolev, V. V. 1960, Moving envelopes of stars (Cambridge, Massachusetts: Harvard University Press)

Toboła, R., Lique, F., Kłos, J., \& Chałasiński, G. 2008, J. Phys. B At. Mol. Phys., 41,155702

van Dishoeck, E. F. 1988, Astrophys. Space Sci. Lib., 146, 49

Velilla Prieto, L., Sánchez Contreras, C., Cernicharo, J., et al. 2017, A\&A, 597, A25

Velilla Prieto, L., Cernicharo, J., Agúndez, M., et al. 2019, A\&A, in press

Winnewisser, G., \& Cook, R. L. 1968, J. Mol. Spectr., 28, 266

Wirsich, J. 1994, ApJ, 424, 370

Yang, X., Chen, P., \& He, J. 2004, A\&A, 414, 1049

Zhang, K., Jiang, B. W., \& Li, A. 2009, ApJ, 702, 680

Zhukovska, S., \& Gail, H.-P. 2008, A\&A, 486, 229 


\section{Appendix A: Additional table}

Table A.1. Observed line parameters of CS $J=3-2$, SiO $J=3-2$, and the $J=7-6$ and $J=8-7$ lines of SiS.

\begin{tabular}{|c|c|c|c|c|}
\hline Line & $\begin{array}{c}v_{\text {calc }} \\
(\mathrm{MHz})\end{array}$ & $\begin{array}{c}v_{\mathrm{obs}} \\
(\mathrm{MHz})\end{array}$ & $\begin{array}{c}V_{\exp } \\
\left(\mathrm{km} \mathrm{s}^{-1}\right)\end{array}$ & $\int_{\left(\mathrm{K} \mathrm{km} \mathrm{s}^{-1}\right)} T_{\mathrm{mb}} \mathrm{d} v$ \\
\hline \multicolumn{5}{|c|}{ IRC +10216 } \\
\hline $\mathrm{SiO} J=3-2$ & 130268.665 & $130268.4(1)$ & 13.1(1) & $130.7(13)$ \\
\hline $\operatorname{SiS} J=7-6$ & 127076.180 & $127076.0(1)$ & $12.7(1)$ & $162.9(16)$ \\
\hline $\operatorname{SiS} J=8-7$ & 145227.054 & $145227.0(1)$ & $14.7(1)$ & $270.5(27)$ \\
\hline $\mathrm{CS} J=3-2$ & 146969.025 & $146968.7(1)$ & $14.7(1)$ & $379.3(37)$ \\
\hline \multicolumn{5}{|c|}{ CIT 6} \\
\hline $\mathrm{SiO} J=3-2$ & 130268.665 & $130267.8(5)$ & $16.2(5)$ & $19.8(19)$ \\
\hline $\operatorname{SiS} J=7-6$ & 127076.180 & $127075.3(5)$ & $16.4(5)$ & $7.55(7)$ \\
\hline $\operatorname{SiS} J=8-7$ & 145227.054 & $145225.4(10)$ & $17.5(10)$ & $12.5(19)$ \\
\hline $\mathrm{CS} J=3-2$ & 146969.025 & $146968.6(5)$ & $16.4(5)$ & $89.0(89)$ \\
\hline \multicolumn{5}{|c|}{ CRL 3068} \\
\hline $\mathrm{SiO} J=3-2$ & 130268.665 & $130268.4(1)$ & $12.3(1)$ & $2.18(2)$ \\
\hline $\operatorname{SiS} J=7-6$ & 127076.180 & $127075.9(1)$ & $12.2(1)$ & $6.36(5)$ \\
\hline $\operatorname{SiS} J=8-7$ & 145227.054 & $145226.7(1)$ & 13.8(1) & $9.24(9)$ \\
\hline $\mathrm{CS} J=3-2$ & 146969.025 & $146968.6(1)$ & $14.2(1)$ & $17.2(10)$ \\
\hline \multicolumn{5}{|c|}{ S Cep } \\
\hline $\mathrm{SiO} J=3-2$ & 130268.665 & $130268.6(1)$ & $23.3(1)$ & $7.80(7)$ \\
\hline $\operatorname{SiS} J=7-6$ & 127076.180 & $127076.5(10)$ & $21.0(5)$ & $0.30(6)^{(a)}$ \\
\hline $\operatorname{SiS} J=8-7$ & 145227.054 & $145227.2(10)$ & $20.8(10)$ & $0.34(7)^{(a)}$ \\
\hline $\operatorname{CS} J=3-2$ & 146969.025 & $146968.9(1)$ & $23.8(1)$ & $19.8(2)$ \\
\hline \multicolumn{5}{|c|}{ IRC +30374 } \\
\hline $\mathrm{SiO} J=3-2$ & 130268.665 & $130268.7(1)$ & $25.1(2)$ & $7.07(7)$ \\
\hline $\operatorname{SiS} J=7-6$ & 127076.180 & $127076.3(1)$ & $22.6(4)$ & $1.73(2)$ \\
\hline $\operatorname{SiS} J=8-7$ & 145227.054 & $145227.2(1)$ & $25.2(2)$ & $2.99(3)$ \\
\hline $\mathrm{CS} J=3-2$ & 146969.025 & $146968.6(1)$ & $25.8(2)$ & $26.7(26)$ \\
\hline \multicolumn{5}{|c|}{ LP And } \\
\hline $\mathrm{SiO} J=3-2$ & 130268.665 & $130268.5(1)$ & $12.9(2)$ & $8.56(8)$ \\
\hline $\operatorname{SiS} J=7-6$ & 127076.180 & 127 076.1(1) & $12.1(2)$ & $7.01(7)$ \\
\hline $\operatorname{SiS} J=8-7$ & 145227.054 & $145226.9(1)$ & $13.8(1)$ & $12.3(1)$ \\
\hline $\mathrm{CS} J=3-2$ & 146969.025 & $146969.0(1)$ & $14.7(1)$ & $35.6(35)$ \\
\hline \multicolumn{5}{|c|}{ V Cyg } \\
\hline $\mathrm{SiO} J=3-2$ & 130268.665 & $130268.5(1)$ & $12.2(1)$ & $9.03(9)$ \\
\hline $\operatorname{SiS} J=7-6$ & 127076.180 & $127076.4(1)$ & $12.9(1)$ & $1.41(1)$ \\
\hline $\operatorname{SiS} J=8-7$ & 145227.054 & $145227.2(1)$ & $12.3(1)$ & $2.45(2)$ \\
\hline $\mathrm{CS} J=3-2$ & 146969.025 & $146968.8(1)$ & $12.1(1)$ & $25.2(25)$ \\
\hline \multicolumn{5}{|c|}{ V384 Per } \\
\hline $\mathrm{SiO} J=3-2$ & 130268.665 & $130268.6(1)$ & $14.8(1)$ & $9.29(9)$ \\
\hline $\operatorname{SiS} J=7-6$ & 127076.180 & $127076.1(1)$ & $12.7(1)$ & $2.15(2)$ \\
\hline $\operatorname{SiS} J=8-7$ & 145227.054 & $145227.0(1)$ & 14.1(1) & $4.02(4)$ \\
\hline $\mathrm{CS} J=3-2$ & 146969.025 & $146968.8(1)$ & $15.6(1)$ & $28.7(29)$ \\
\hline \multicolumn{5}{|c|}{ IRC +60144 } \\
\hline $\mathrm{SiO} J=3-2$ & 130268.665 & $130268.6(1)$ & $20.8(1)$ & $5.34(5)$ \\
\hline $\operatorname{SiS} J=7-6$ & 127076.180 & $127076.2(10)$ & $19.4(10)$ & $0.9(2)^{(a)}$ \\
\hline $\operatorname{SiS} J=8-7$ & 145227.054 & $145226.9(1)$ & $20.9(1)$ & $1.65(1)$ \\
\hline $\mathrm{CS} J=3-2$ & 146969.025 & $146968.7(1)$ & $20.9(1)$ & $12.5(12)$ \\
\hline
\end{tabular}

Notes. Numbers in parentheses are $1 \sigma$ uncertainties in units of the last digits. ${ }^{(a)}$ Marginal detection. 
Table A.1. continued.

\begin{tabular}{|c|c|c|c|c|}
\hline Line & $\begin{array}{c}v_{\text {calc }} \\
(\mathrm{MHz})\end{array}$ & $\begin{array}{c}v_{\mathrm{obs}} \\
(\mathrm{MHz})\end{array}$ & $\begin{array}{c}V_{\exp } \\
\left(\mathrm{km} \mathrm{s}^{-1}\right)\end{array}$ & $\underset{\left(\mathrm{K} \mathrm{km} \mathrm{s}^{-1}\right)}{\int_{\mathrm{mb}} \mathrm{d} v}$ \\
\hline \multicolumn{5}{|c|}{ U Cam } \\
\hline $\mathrm{SiO} J=3-2$ & 130268.665 & $130268.2(1)$ & $11.6(1)$ & $0.96(1)$ \\
\hline $\operatorname{SiS} J=7-6$ & 127076.180 & - & - & - \\
\hline $\operatorname{SiS} J=8-7$ & 145227.054 & - & - & - \\
\hline $\mathrm{CS} J=3-2$ & 146969.025 & $146968.8(1)$ & $13.5(1)$ & $4.7(5)$ \\
\hline \multicolumn{5}{|c|}{ IRC +20370} \\
\hline $\mathrm{SiO} J=3-2$ & 130268.665 & $130268.5(1)$ & $13.1(1)$ & $7.25(7)$ \\
\hline $\operatorname{SiS} J=7-6$ & 127076.180 & $127076.1(1)$ & $12.7(1)$ & $3.10(2)$ \\
\hline $\operatorname{SiS} J=8-7$ & 145227.054 & $145227.1(5)$ & $13.8(4)$ & $5.64(5)$ \\
\hline $\mathrm{CS} J=3-2$ & 146969.025 & $146968.9(5)$ & $13.2(8)$ & $19.9(20)$ \\
\hline \multicolumn{5}{|c|}{ CRL 67} \\
\hline $\mathrm{SiO} J=3-2$ & 130268.665 & $130268.6(1)$ & $14.5(1)$ & $2.88(3)$ \\
\hline $\operatorname{SiS} J=7-6$ & 127076.180 & 127 076.4(1) & $13.6(1)$ & $1.62(1)$ \\
\hline $\operatorname{SiS} J=8-7$ & 145227.054 & $145226.9(1)$ & $15.4(2)$ & $2.83(2)$ \\
\hline $\mathrm{CS} J=3-2$ & 146969.025 & $146968.9(1)$ & $16.1(2)$ & $12.3(12)$ \\
\hline \multicolumn{5}{|c|}{ CRL 190} \\
\hline $\mathrm{SiO} J=3-2$ & 130268.665 & $130269.5(5)$ & $16.3(5)$ & $0.39(4)$ \\
\hline $\operatorname{SiS} J=7-6$ & 127076.180 & $127076.2(1)$ & $16.6(1)$ & $1.45(1)$ \\
\hline $\operatorname{SiS} J=8-7$ & 145227.054 & $145227.0(1)$ & $16.2(1)$ & $1.93(2)$ \\
\hline $\mathrm{CS} J=3-2$ & 146969.025 & $146968.7(1)$ & $16.9(2)$ & $6.76(7)$ \\
\hline \multicolumn{5}{|c|}{ V Aql } \\
\hline $\mathrm{SiO} J=3-2$ & 130268.665 & $130268.4(1)$ & $7.2(1)$ & $0.44(4)$ \\
\hline $\operatorname{SiS} J=7-6$ & 127076.180 & - & - & - \\
\hline SiS $J=8-7$ & 145227.054 & - & - & - \\
\hline $\operatorname{CS} J=3-2$ & 146969.025 & $146968.8(1)$ & $9.2(1)$ & $4.30(4)$ \\
\hline \multicolumn{5}{|c|}{ CRL 2477} \\
\hline $\mathrm{SiO} J=3-2$ & 130268.665 & $130268.1(1)$ & $16.7(1)$ & $1.28(1)$ \\
\hline $\operatorname{SiS} J=7-6$ & 127076.180 & $127076.2(1)$ & $16.5(1)$ & $2.64(2)$ \\
\hline $\operatorname{SiS} J=8-7$ & 145227.054 & $145226.8(1)$ & $19.2(2)$ & $2.93(3)$ \\
\hline $\operatorname{CS} J=3-2$ & 146969.025 & $146968.6(1)$ & 20.1(1) & $7.17(7)$ \\
\hline \multicolumn{5}{|c|}{ CRL 2494} \\
\hline $\mathrm{SiO} J=3-2$ & 130268.665 & $130268.8(1)$ & $16.4(2)$ & $2.64(3)$ \\
\hline $\operatorname{SiS} J=7-6$ & 127076.180 & $127077.0(10)$ & $20.4(10)$ & $1.04(20)$ \\
\hline $\operatorname{SiS} J=8-7$ & 145227.054 & $145227.4(2)$ & $19.0(6)$ & $1.57(15)$ \\
\hline $\operatorname{CS} J=3-2$ & 146969.025 & $146968.9(1)$ & $19.6(1)$ & $13.2(13)$ \\
\hline \multicolumn{5}{|c|}{ Rv Aqr } \\
\hline $\mathrm{SiO} J=3-2$ & 130268.665 & $130268.3(1)$ & $14.0(2)$ & $5.58(5)$ \\
\hline $\operatorname{SiS} J=7-6$ & 127076.180 & $127076.1(5)$ & $13.3(5)$ & $0.71(7)$ \\
\hline $\operatorname{SiS} J=8-7$ & 145227.054 & $145226.9(1)$ & $14.6(2)$ & $1.34(1)$ \\
\hline $\mathrm{CS} J=3-2$ & 146969.025 & $146968.6(1)$ & $15.4(2)$ & $13.3(13)$ \\
\hline \multicolumn{5}{|c|}{ CRL 2513} \\
\hline $\mathrm{SiO} J=3-2$ & 130268.665 & $130268.5(1)$ & $25.7(1)$ & $2.58(2)$ \\
\hline $\operatorname{SiS} J=7-6$ & 127076.180 & $127076.0(5)$ & $24.2(6)$ & $0.84(8)$ \\
\hline $\operatorname{SiS} J=8-7$ & 145227.054 & $145226.7(5)$ & $24.9(4)$ & $1.66(16)$ \\
\hline $\mathrm{CS} J=3-2$ & 146969.025 & $146968.7(1)$ & $26.5(2)$ & $8.92(9)$ \\
\hline \multicolumn{5}{|c|}{ S Aur } \\
\hline $\mathrm{SiO} J=3-2$ & 130268.665 & $130267.5(1)$ & $21.8(2)$ & $1.25(1)$ \\
\hline $\operatorname{SiS} J=7-6$ & 127076.180 & - & - & - \\
\hline $\operatorname{SiS} J=8-7$ & 145227.054 & - & - & - \\
\hline $\mathrm{CS} J=3-2$ & 146969.025 & $146968.1(1)$ & $26.5(1)$ & $4.05(4)$ \\
\hline
\end{tabular}


Table A.1. continued.

\begin{tabular}{|c|c|c|c|c|}
\hline Line & $\begin{array}{c}v_{\text {calc }} \\
(\mathrm{MHz})\end{array}$ & $\begin{array}{c}v_{\mathrm{obs}} \\
(\mathrm{MHz})\end{array}$ & $\begin{array}{c}V_{\exp } \\
\left(\mathrm{km} \mathrm{s}^{-1}\right)\end{array}$ & $\underset{\left.(\mathrm{K} \mathrm{km} \mathrm{s})^{-1}\right)}{\int_{\mathrm{mb}} \mathrm{d} v}$ \\
\hline \multicolumn{5}{|c|}{ V636 Mon } \\
\hline $\operatorname{SiO} J=3-2$ & 130268.665 & $130269.3(1)$ & $24.3(1)$ & $3.74(4)$ \\
\hline $\operatorname{SiS} J=7-6$ & 127076.180 & $127076.2(5)$ & $24.1(5)$ & $0.60^{(a)}$ \\
\hline $\operatorname{SiS} J=8-7$ & 145227.054 & $145227.9(5)$ & $26.5(5)$ & $0.50(5)^{(a)}$ \\
\hline CS $J=3-2$ & 146969.025 & $146969.8(1)$ & 25.8(1) & $8.85(9)$ \\
\hline \multicolumn{5}{|c|}{ W Ori } \\
\hline $\mathrm{SiO} J=3-2$ & 130268.665 & $130268.1(5)$ & $8.5(4)$ & $0.29(3)$ \\
\hline $\operatorname{SiS} J=7-6$ & 127076.180 & - & - & - \\
\hline $\operatorname{SiS} J=8-7$ & 145227.054 & - & - & - \\
\hline CS $J=3-2$ & 146969.025 & $146968.8(5)$ & $10.5(8)$ & $4.2(4)$ \\
\hline \multicolumn{5}{|c|}{ YCVn } \\
\hline $\mathrm{SiO} J=3-2$ & 130268.665 & $130268.3(1)$ & $7.3(1)$ & $0.38(4)$ \\
\hline $\operatorname{SiS} J=7-6$ & 127076.180 & - & - & - \\
\hline $\operatorname{SiS} J=8-7$ & 145227.054 & - & - & - \\
\hline $\mathrm{CS} J=3-2$ & 146969.025 & $146968.5(1)$ & $9.4(1)$ & $7.58(7)$ \\
\hline \multicolumn{5}{|c|}{ R Lep } \\
\hline $\mathrm{SiO} J=3-2$ & 130268.665 & $130267.9(1)$ & $19.8(2)$ & $3.45(3)$ \\
\hline $\operatorname{SiS} J=7-6$ & 127076.180 & - & - & - \\
\hline $\operatorname{SiS} J=8-7$ & 145227.054 & - & - & \\
\hline CS $J=3-2$ & 146969.025 & $146968.3(1)$ & $20.9(2)$ & $5.78(6)$ \\
\hline \multicolumn{5}{|c|}{ ST Cam } \\
\hline $\operatorname{SiO} J=3-2$ & 130268.665 & - & - & - \\
\hline $\operatorname{SiS} J=7-6$ & 127076.180 & - & - & - \\
\hline $\operatorname{SiS} J=8-7$ & 145227.054 & - & - & - \\
\hline $\mathrm{CS} J=3-2$ & 146969.025 & $146969.2(1)$ & $11.5(1)$ & $0.82(8)$ \\
\hline \multicolumn{5}{|c|}{ UU Aur } \\
\hline $\operatorname{SiO} J=3-2$ & 130268.665 & $130266.2(10)$ & $6.7(10)$ & $0.09(2)$ \\
\hline $\operatorname{SiS} J=7-6$ & 127076.180 & - & - & - \\
\hline $\operatorname{SiS} J=8-7$ & 145227.054 & - & - & - \\
\hline $\mathrm{CS} J=3-2$ & 146969.025 & $146968.6(10)$ & $11.0(1)$ & $0.30(3)$ \\
\hline
\end{tabular}

